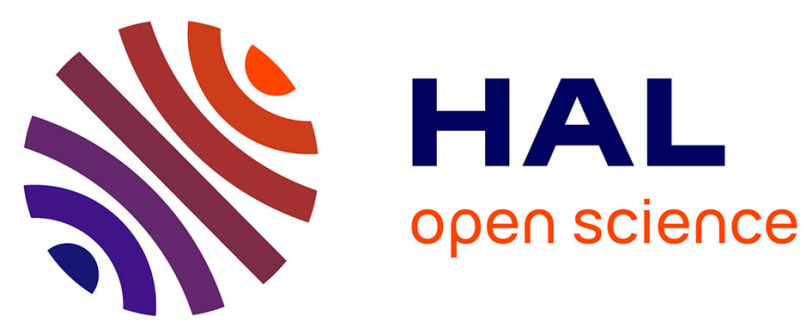

\title{
Structure and Charge Heterogeneity in Isomeric Au25(MBA)18 Nanoclusters-Insights from Ion Mobility and Mass Spectrometry
}

Clothilde Comby-Zerbino, Franck Bertorelle, Philippe Dugourd, Rodolphe Antoine, Fabien Chirot

\section{To cite this version:}

Clothilde Comby-Zerbino, Franck Bertorelle, Philippe Dugourd, Rodolphe Antoine, Fabien Chirot. Structure and Charge Heterogeneity in Isomeric Au25(MBA)18 Nanoclusters-Insights from Ion Mobility and Mass Spectrometry. Journal of Physical Chemistry A, 2020, 124 (28), pp.5840-5848. 10.1021/acs.jpca.0c03131 . hal-02919814

\section{HAL Id: hal-02919814 https://hal.science/hal-02919814}

Submitted on 31 Oct 2020

HAL is a multi-disciplinary open access archive for the deposit and dissemination of scientific research documents, whether they are published or not. The documents may come from teaching and research institutions in France or abroad, or from public or private research centers.
L'archive ouverte pluridisciplinaire HAL, est destinée au dépôt et à la diffusion de documents scientifiques de niveau recherche, publiés ou non, émanant des établissements d'enseignement et de recherche français ou étrangers, des laboratoires publics ou privés. 


\section{Structure and charge heterogeneity in isomeric $\mathrm{Au}_{25}(\mathrm{MBA})_{18}$ nanoclusters - insights from ion mobility and mass spectrometry}

Clothilde Comby-Zerbino, ${ }^{1}$ Franck Bertorelle, ${ }^{1}$ Philippe Dugourd, ${ }^{1}$ Rodolphe Antoine, ${ }^{* 1}$ and Fabien Chirot*2

${ }^{1}$ Univ Lyon, Université Claude Bernard Lyon 1, CNRS, UMR5306 Institut Lumière Matière, F-69100, 5 rue de la Doua, VILLEURBANNE, France

¿Univ Lyon, Université Claude Bernard Lyon 1, CNRS, UMR5280 Institut des Sciences Analytiques, F-69100, 5 rue de la Doua, VILLEURBANNE, France

$\underline{* \text { Contact: }} \underline{\text { fabien.chirot@univ-lyon1.fr, rodolphe.antoine@univ-lyon1.fr }}$

\section{Abstract:}

Atomically precise $\mathrm{Au}_{25}(\mathrm{MBA})_{18}$ nanoclusters were investigated by mass spectrometry and ion mobility spectrometry. We show that clusters sharing the same chemical composition and bearing the same net charge may display different structures and different charge repartition patterns, in relation with different deprotonation states of the ligands. Part of the observed heterogeneity is a consequence of spontaneous electron loss occurring in the gas phase, which modifies the net charge of the clusters, while maintaining the initial protonation state. 


\section{Introduction}

The physico-chemical properties of small metal nanoclusters, composed of less than a hundred atoms, dramatically depend on their composition and structure. Different wet chemistry synthesis strategies have been developed to precisely control those parameters at the atomic level, and then design species with tailored properties. Such strategies have been especially fruitful to obtain gold nanoclusters stabilized by thiolated ligands (SR), yielding species of general composition $\left(A u_{n} \mathrm{SR}_{m}\right) \cdot{ }^{1-4}$ Among these nanoclusters, $\mathrm{Au}_{25} \mathrm{SR}_{18}$ has a special place due to its high stability and its ease of preparation and functionalization. ${ }^{5-7}$ Depending on the conditions of the preparation and storage, various charge states of $\mathrm{Au}_{25} \mathrm{SR}_{18}$ have been figured out (e.g. $\left.\left[\mathrm{Au}_{25} \mathrm{SR}_{18}\right]^{-1,0+1}\right) \cdot{ }^{8-10} \quad \mathrm{Au}_{25} \mathrm{SR}_{18}$ nanoclusters with different charge states possess diverse properties including magnetism, ${ }^{10,11}$ optical absorption, ${ }^{8,9}$ catalytic activity ${ }^{12}$ and stability. ${ }^{13,14}$ Furthermore, Maran and co-workers studied the reduction or oxidation of $\left[\mathrm{Au}_{25} \mathrm{SR}_{18}\right]^{0}$ and evidenced a series of charge states, $-2,-1,+1,+2$, and +3 , under cyclic voltammetry. ${ }^{15}$

Whetten, Tsukuda and coworkers further investigated the effect of charge on the stability of $\mathrm{Au}_{25} \mathrm{SR}_{18}{ }^{8}$ By judiciously chosing organic ligands lacking protonation or deprotonation sites, they managed to change the net charge on the cluster the core, while preserving the magic stability of the cluster. ${ }^{16-18}$ These results suggest that the magic stability of $\mathrm{Au}_{25}(\mathrm{SR})_{18}$, is attributable to geometric rather than electronic factors. Clearly, electrospray ionization mass spectrometry (ESI-MS) offered the opportunity to accurately map the charge state of $\operatorname{Au}_{25}(\mathrm{SR})_{18}$ nanoclusters. ${ }^{16-20}$ Mass spectrometry analysis of gold clusters after electrospray ionization usually yields ionic species with different charge states, depending on the propensity of the ligand to bear charges, most of the time through protonation or deprotonation. In this case, the observed species are of the form $\left[\mathrm{Au}_{\mathrm{n}} \mathrm{SR}_{\mathrm{n}} \pm \mathrm{mH}\right]^{ \pm \mathrm{z}}$. In such species, part of the charge is borne by ligand moieties, through protonation or deprotonation, but the gold cluster itself can also be charged. Deconvolution of the isotope-resolved mass spectrum can then yield information on the charge state of the cluster, which can be further interpreted in terms of the oxidation state. ${ }^{19}$

Recently, using first principles calculations, the effects of charge state and type of ligands on the structural, electronic, and optical properties of the $\operatorname{Au}_{25}(\mathrm{SR})_{18}$ 
nanocluster were investigated. ${ }^{21}$ It was shown that distortions of the metal core become more pronounced when the charge state of the nanocluster increases (i.e. going from the anionic, to neutral, to cationic cluster) affecting optical spectra.

In addition to mass spectrometry, novel and complementary information on the intrinsic stability and structures of nanoclusters can be obtained by applying gasphase methods, ${ }^{22}$ including ion mobility mass spectrometry. Ion mobility-massspectrometry (IMS-MS) has also been used to gain insight in the structural diversity of nanoclusters, ${ }^{23-28}$ and on the influence of the charge state of cluster ions on their structure. ${ }^{20}$ An advantage of IMS and MS in the present context is their ability to isolate and study separately the different species that may co-exist in the sample.

In the present study, we used high resolution IMS and MS measurements to investigate the charging patterns of gold clusters ligated by mercaptobenzoic acid (MBA). Protons can be removed from MBA carboxyl groups. In the negative mode of ionization, the charging patterns can thus be a combination of the net charge of the core and the charge of the deprotonated ligands. In a previous study, we reported the influence of the ligand structure on the stability of the clusters by comparing $\mathrm{Au}_{25} \mathrm{MBA}_{18}$ clusters composed with different substitution isomers of MBA (ortho, meta, and para). ${ }^{20}$ In the following, we show that deconvolution of the mass spectra obtained for those clusters reveals that different charging patterns co-exist. Based on IMS separation of these populations and IMS-IMS-MS measurements, we give evidence that spontaneous electron loss occurring in the gas phase accounts for a large part of the observed diversity.

\section{Experimental Section}

\section{Materials}

Tetrachloroauric(III) acid trihydrate $\left(\mathrm{HAuCl}_{4} \cdot 3 \mathrm{H}_{2} \mathrm{O}\right)$, 4-mercaptobenzoic acid( $p$ MBA), and 3-mercaptobenzoic acid ( $m$-MBA) were obtained from Acros Organics; thiosalicylic acid (2-mercaptobenzoic acid, o-MBA), tributylamine, and ammonium acetate were purshased from Sigma-Aldrich; borane-trimethylamine complex was obtained from Alfa Aesar; and methanol and diethyl ether were obtained from VWR. Milli-Q water with a resistivity of $18.2 \mathrm{M} \Omega \mathrm{cm}$ was used for all experiments. 


\section{Synthesis of $\mathrm{Au}_{25} \mathrm{MBA}_{18}$ Clusters}

Similar synthetic conditions were used for the three (para, meta, or ortho) clusters. These conditions are thoroughly described in ref. 20. Briefly, mercaptobenzoic acid is dissolved in methanol and tributylamine, and tetrachloroauric acid trihydrate is added to form a gold thiolate/tributylamine complex. A slow reduction of gold is then obtained by adding trimethylamine borane. The mixture is finally left under stirring for $24 \mathrm{~h}$.

\section{IMS-MS and IMS-IMS-MS measurements}

High resolution IMS-MS measurements were done using a home-built IMS-IMSMS setup (see scheme S1 in supporting information) described in details in ref. 29 Solutions of $\mathrm{Au}_{25} \mathrm{MBA}_{18}$ were prepared at a concentration of $50 \mu \mathrm{mol} . \mathrm{L}^{-1}$ in ultrapure water and electrosprayed directly in the negative ion mode. We used the electrospray ion source of a Maxis Impact quadrupole-time-of-flight instrument (Bruker, Bremen, Germany), and the source conditions were adapted to minimize cluster fragmentation (see Supplementary Information). In our instrument, ions cross two identical drift tubes before being detected as a function of their massto-charge $(\mathrm{m} / \mathrm{z})$ ratio by a time-of-flight mass spectrometer (Maxis Impact Bruker, Bremen, Germany). This configuration allows to record mass spectra as a function of the drift time of the ions across the drift tubes. Arrival time distributions (ATDs) can then be extracted for species in $\mathrm{m} / \mathrm{z}$ ranges of interest. The arrival time of the ions depends on the ratio of their orientationally-averaged collision integral with the drift gas (or CCS), $\Omega$, and their charge $q::^{30}$

$$
t_{d}=\frac{16}{3} L \sqrt{\mu \frac{k_{B} T}{2 \pi}} \frac{N}{E} \frac{\Omega}{q}
$$

where $t_{d}$ is the time through a drift region of length $L, k_{B}$ is the Boltzmann constant, $T$ is the drift temperature, $\mathrm{E}$ is the drift field, $N$ is the number density of the buffer gas, and $\mu$ is the reduced mass for ion-neutral collisions.

The two drift tubes, DT1 and DT2, are $79 \mathrm{~cm}$ long. For the present experiments, the drift region was filled with 4 Torr helium maintained at room temperature (298 K). A constant DC electric field of $6.3 \mathrm{~V} \cdot \mathrm{cm}^{-1}$ was applied across each tube. As depicted in Scheme S1, three dual ion funnel assemblies, FA, FB, and FC separate the different regions of the instrument. They are used to ensure for good 
transmission of the ions. FA and FB also serve as ion traps and can be used to inject short (300 $\mu \mathrm{s}$ ) ion bunches in DT1 or DT2, respectively, thus defining a reference for $\mathrm{t}=0$ in IMS arrival time measurements. This dual tube configuration allows different operation modes.

\section{IMS measurements}

For high-resolution IMS measurements, ion bunches are injected from FA and a DC gradient (typically 6-8 V.cm-1) is applied across FB to prevent ions from being trapped in this region. Arrival times are then measured using DT1 and DT2 as a single drift tube. This mode allows to achieve high IMS resolution by taking advantage of a longer drift path, and a higher drift voltage. The resolving power for IMS separation is limited by diffusion and scales with the square root of the drift voltage as: ${ }^{30}$

$$
R_{\text {diff }}=\frac{t_{d}}{\Delta t_{d}}=\frac{1}{4 \sqrt{\ln (2)}} \sqrt{\frac{q V}{k_{B} T}}
$$

where $\Delta t_{d}$ is the FWHM of a diffusion-limited IMS peak, $k_{B}$ is the Boltzmann constant, $T$ is the drift temperature, $q$ is the charge of the ions, and $V$ is the voltage across the drift cell. In the present experimental conditions, using drift voltage of $1000 \mathrm{~V}$ across DT1+DT2, the theoretical resolving power for ions with 5 charges is then $R_{\text {diff }}=132$. In practice, the finite duration of the injected ion bunch limits the effective experimental resolving power to 80-90 for the ions of interest. In the experimental ATDs recorded in this operation mode, the arrival time refers to the injection in DT1.

\section{IMS-IMS measurements}

In the IMS-IMS operation mode, the ion gate at the end of DT1 is used to cut a "slice" in the ATD, thus letting through only the ions in a narrow arrival time range. This allows isomer selection. ${ }^{31-33}$ The ATDs recorded in this mode gives information about potential modifications of the ions after selection. Namely, an ATD not consisting in a single peak centered at the selected drift time provides a signature of a change in the mobility of the ions, i.e. a change in their "surface-to-charge" ratio. This procedure was previously used to study isomerization, corresponding to a change of CCS, ${ }^{34,35}$ but it is also clear from equation (1), that a change in the charge $q$ also affects the arrival time. 


\section{MS-MS measurements}

Classical collision induced dissociation experiments were performed using the quadrupole of the Maxis Impact to select precursor ions, and the time-of-flight to detect fragments. Selected ions were subjected to collisional activation by varying the relative bias voltage of the quadrupole and the collision cell. We used dry $\mathrm{N}_{2}$ as a collision gas. The following instrument settings were used (as defined in Bruker o-tofControl software): collision gas flow: $22 \%$, quadrupole isolation width: $\pm 5 \mathrm{~m} / \mathrm{z}$.

\section{Deconvolution of the mass spectra}

The isotope distributions of the ions of interest were fitted using homemade python software based on the SciPy library (version 1.2.1). ${ }^{36}$ Our goal was to determine the relative populations of species with close chemical compositions (typically differing by one or two protons), thus displaying overlapping isotope distributions. The molmass package ${ }^{37}$ was used to compute theoretical isotope distributions for the different considered compositions. Theoretical isotopic distributions for the mixture were generated from a linear combination of the isotope distributions of the different considered compositions. An isotope pattern with Gaussian peaks was then computed from those distributions. This simulated pattern was finally adjusted to the experimental data, through minimization of the RMSD, leaving the weights of the different populations as only free fitting parameters. These weights were then interpreted as the relative populations of the different species detected for each charge state.

It important to note that the masses of the different candidate compositions are shifted by only one mass unit. The intensity of the peak at the lowest $\mathrm{m} / \mathrm{z}$ value then only corresponds to the species with the lowest mass (this is the monoisotopic peak for this species). We took advantage of this particularity by using a stepwise fitting procedure. The weight for the lowest mass species was first adjusted to fit the first mass peak. Then the weight for the second lowest mass species was adjusted to fit the second mass peak, keeping the first weight constant, etc.

\section{Results and Discussion}

Different populations with distinct charging patterns co-exist. Mass spectra were recorded in the negative electrospray mode for $\mathrm{Au}_{25} \mathrm{MBA}_{18}$ samples prepared 
with MBA ligands substituded either in ortho, meta, or para position. As previously reported, ${ }^{20}$ these spectra are dominated by peaks corresponding to the different charge states of $\mathrm{Au}_{25} \mathrm{MBA}_{18}$. For the three isomers of mercaptobenzoic acids (para/meta/ortho-MBA), the observed charge states range from $z=-3$ to $z=-5$ (data not shown). Higher charge states are nevertheless observed for para-MBA and, to a lesser extent, for meta-MBA containing clusters. ${ }^{20}$

We focused on the isotope distribution on the mass peaks of the different charge states of $\mathrm{Au}_{25} \mathrm{MBA}_{18}$ clusters. Figure 1 displays the isotope pattern for the 5- charge state of $\mathrm{Au}_{25}$ ortho- $\mathrm{MBA}_{18}$ clusters. The observed proton distribution could not be reproduced by considering a unique number $\mathrm{p}$ of deprotonation, using a raw formula of the form $\left[\mathrm{Au}_{25} \text { ortho- } \mathrm{MBA}_{18}-\mathrm{nH}\right]^{-5}$. Namely, the peak at the lowest $\mathrm{m} / \mathrm{z}$ in the pattern corresponds to the monoisotopic mass expected for $\left[\mathrm{Au}_{25}\right.$ ortho$\left.\mathrm{MBA}_{18}-6 \mathrm{H}\right]^{-5}$ ions. However, the intensity ratio of the different peaks of the isotope pattern are not well reproduced based on this composition. Using the abovedescribed fitting procedure, we determined that the observed isotope pattern was resulting from the coexistence of species with different raw formulas, including $\left[\mathrm{Au}_{25} \text { ortho- } \mathrm{MBA}_{18}-6 \mathrm{H}\right]^{-5}$, but also $\left[\mathrm{Au}_{25} \text { ortho- } \mathrm{MBA}_{18}-5 \mathrm{H}\right]^{-5}$ and $\left[\mathrm{Au}_{25}\right.$ ortho$\left.\mathrm{MBA}_{18}-4 \mathrm{H}\right]^{-5}$ in respective proportions of $51.0 \%, 30.9 \%$, and $18.1 \%$. The same deconvolution procedure was applied to all detected charge states for all investigated species, always yielding combinations of populations with different protonation states (see Figure S1).

In negative electrospray mode, deprotonation is a standard way of formation of charged species. In the present clusters, the carboxyl groups of the 18 MBA ligands are the most obvious deprotonation sites. It then seems reasonable to assume that a species with $n$ missing protons can accommodate $n$ charges on deprotonated carboxylate groups. For a given charge state $z$, the present deconvolution yields species of general formula $\left[\mathrm{Au}_{25} \text { ortho- } \mathrm{MBA}_{18}-\mathrm{nH}\right]^{-z}$. If $n \neq z$, only part of the total charge can be attributed to the deprotonated carboxylate groups. In the following, we characterize the difference between the number of deprotonations and the total net charge by defining the charge shift $x=n-z$. This charge shift can be positive, negative or 0 . When it is not 0 , it corresponds to the amount of positive or negative charge which is not a priori borne by a deprotonated carboxylate group. This charge has either to be located in another region of the ligand, at the interface between the gold core and the ligands, at the cluster core, or delocalized. As 
depicted in the inset of Figure 2, for species whose composition follows the raw formula $\left[\mathrm{Au}_{25} \mathrm{MBA}_{18}-(\mathrm{z}+\mathrm{x}) \mathrm{H}\right]^{-z}$ the value of $\mathrm{x}$ completely defines the corresponding charge repartition scheme: $z+x$ negative charges borne by ligand carboxyl groups, and $x$ charges located elsewhere inside the cluster.

As summarized in the pie charts of Figure 2, populations displaying different charge shift, and then different charge repartition schemes, co-exist for all investigated clusters. Their relative proportion varies depending on the substitution of the ligands, and on the net charge state $z$ of the species. For the lowest detected charge states, the observed species mostly correspond to $x=-1$, as already reported.20 As charge state increases however, contributions from other populations become more significant. Interestingly, the evolution of the populations in not smooth but stepwise. In a first step, the population for $x=-1$ decreases to about $50 \%$ of the total ions. Then, for the highest observed charge state, $x=0$, or $x=+1$ dominate, and the proportion of $x=-1$ is dramatically reduced. This two-step evolution is observed for the three isomers of mercaptobenzoic acids, but the charge state at which the steps occur are different. Namely, for ortho-MBA only three charge states are observed showing significantly different populations. In contrast, the populations for para-MBA remain similar up to charge state $z=-5$, and up to $z=-4$ for meta-MBA. To summarize, significant changes in the charge repartition scheme are observed only for the highest two charge states detected for each isomeric species.

The stability of the clusters is affected by their charging pattern. Previously reported CID experiments on the 4- charge states of $\mathrm{Au}_{25} \mathrm{MBA}_{18}$ clusters emphasized the influence of the substitution of the MBA ligand on the stability of the clusters. Namely, the relative stabilities of the three considered species was found to be para>meta>ortho. ${ }^{20}$ In order to investigate the influence of the charging pattern on the stability of the clusters, we performed CID experiments. We focused on the 5- charge state of the investigated clusters, because it corresponds to a broad diversity of populations and it is detected for all ligand substitutions. The evolution of the intensity of the detected fragments are plotted in Figure S2. Common fragmentation channels shared by the three considered ligand geometries include the apparition of $\left[\mathrm{Au}_{2} \mathrm{MBA}_{2}\right]^{-1}$, and the loss of $\left[\mathrm{AuMBA}{ }_{2}\right]^{-}$ ${ }^{1}$ for the parent precursors. As already reported for the triply charged ions, clusters with ortho-MBA appear to fragment at significantly lower voltages that the other 
two. As a consequence, more species can be identified in the fragment mass spectra for these clusters, which can be attributed to the loss of up to two gold atoms, and up to five ligands. ${ }^{20}$

Based on the above-described deconvolution method, we extracted the evolution of the different populations identified within the precursor ion peak (e.g. $\left[\mathrm{Au}_{25}\right.$ $\left.\mathrm{MBA}_{18}-\mathrm{nH}\right]^{-z}$ with $\mathrm{x}=-1,0$ and +1 ) as a function of the collision voltage. The results are displayed on Figure 3. For clusters with para- and meta-MBA, no evolution is observed below a threshold voltage of $5 \mathrm{~V}$ and $9 \mathrm{~V}$ for meta- and para-, respectively. In contrast, the populations of clusters with ortho-MBA are modified yet at the lowest collision voltages, in relation with a decreased stability (see above). Once fragmentation is observed, the relative populations of species with different charge shifts are modified in each case. For meta- and para-substituted species, the populations corresponding to $\mathrm{x}=-1$ are decreased relative to the others. This is also true for clusters with ortho-MBA, but a less pronounced decrease is also observed for the $x=0$ species. Interpreting those evolutions in terms of relative stability yields the conclusion that a more negative charge shift decreases the overall stability of the clusters.

\section{Evidence for heterogeneity within clusters with similar charging patterns.}

High-resolution arrival time distributions (ATDs) extracted for all detected species are represented in Figure 4. Most ATDs are dominated by a single spectral feature. However, the distributions for $\left[\mathrm{Au}_{25} \text { ortho-MBA}{ }_{18}\right]^{-5},\left[\mathrm{Au}_{25} \mathrm{meta}-\mathrm{MBA}_{18}\right]^{-5}$, and $\left[\mathrm{Au}_{25} \text { para-MBA}{ }_{18}\right]^{-6}$ clearly display two resolved peaks with comparable intensity. The width of these peaks correspond to the resolving power of the instrument (see Figure S3). CCS for charge states 3-, 4- and 5- were reported in ref. 20. In this work, we improved the resolution for CCS measurements, allowing for 2 different CCS values for $\left.\left[\mathrm{Au}_{25} \text { ortho-MBA}\right]_{18}\right]^{-5},\left[\mathrm{Au}_{25} \text { meta-MBA}{ }_{18}\right]^{-5}$, and $\left[\mathrm{Au}_{25} \text { para-MBA }{ }_{18}\right]^{-6}$ and we extended CCS measurements to charge states 6- and 7- (see Table S1 and Figure S4). We observe an increase of the CCS with charge state for clusters with para- and meta-MBA, which can be interpreted as a change in the ligand packing to accommodate Coulomb repulsion. No such change is observed for ortho-MBA, which reveals lower flexibility.

Beyond the most intense peaks, additional spectral features are also visible in most ATDs (see Figure 4). Firstly, the main peaks display a marked shoulder on the high drift time side in ATDs recorded for $\left[\mathrm{Au}_{25} \text { ortho-MBA}{ }_{18}\right]^{-5},\left[\mathrm{Au}_{25} \text { meta-MBA}{ }_{18}\right]^{-5}$, 
$\left[\mathrm{Au}_{25} \text { meta-MBA }{ }_{18}\right]^{-6}$ and $\left[\mathrm{Au}_{25} \text { para-MBA }{ }_{18}\right]^{-6}$. Secondly, a long tail to the short drift time side is also observed for $\left[\mathrm{Au}_{25} \text { ortho-MBA }{ }_{18}\right]^{-4},\left[\mathrm{Au}_{25} \text { meta-MBA }{ }_{18}\right]^{-4},\left[\mathrm{Au}_{25}\right.$ para$\left.\mathrm{MBA}_{18}\right]^{-5}$. In order to interpret these different spectral features, we took advantage of the possibility to extract from our raw data either the ATD for a single isotopic species, or conversely the mass spectrum corresponding to a selected arrival time range. On the left panel of Figure 5, we plotted the ATD for the peak at $\mathrm{m} / \mathrm{z} 1534.6$, i.e. the monoisotopic peak of $\left[\mathrm{Au}_{25} \text { meta-MBA } \mathrm{Ar}_{18}-5 \mathrm{H}\right]^{-5}(\mathrm{x}=0)$. This monoisotopic ATD significantly differs from the full ATD obtained by integrating the whole isotopic pattern around $\mathrm{m} / \mathrm{z} 1535$, since the peak at $26.6 \mathrm{~ms}$ is not present. This observation suggests that the different features in the ATD may correspond to populations with different charging patterns. This is supported by the mass spectra on the right panel of Figure 5, which were obtained by integrating over different arrival time windows corresponding to the main spectral features observed in the full ATD, and denoted A, B, and $C$, in order of their respective arrival time. As expected from above, the extracted mass spectrum for peak $B$ reasonably matches with the expected isotope distribution for $\left[\mathrm{Au}_{25}\right.$ meta-MBA ${ }_{18}$ $5 \mathrm{H}]^{-5}(x=0)$. Integrating across peak $A$ yields a mass spectrum shifted to higher $\mathrm{m} / \mathrm{z}$, which corresponds to a majority of $\left[\mathrm{Au}_{25} \text { meta-MBA}{ }_{18}-4 \mathrm{H}\right]^{-5}(\mathrm{x}=-1)$. We interpret the minor peak at $\mathrm{m} / \mathrm{z} 1534.6$ in the projected mass spectrum for $A$ to partial overlap with peak $B$. The isotope pattern obtained for peak $C$ is more complex and has to be considered as resulting from the coexistence of different populations with different charge shifts ranging from -1 to +1 . Similar observations can be done from the deconvolution of the other multimodal ATDs (Figure S5). Interestingly, different populations with the same charge shift can be found at significantly different arrival times. This suggests that, beyond the heterogeneity of the clusters in terms of charging patterns, cluster with the same extra charge can also have different mobilities, and then possibly different structures. The existence of structural heterogeneity, in relation with different distributions of the net charge, was indeed recently predicted from first principles calculations. ${ }^{21,38,39}$

\section{Deconvolution of the ATDs reveals that clusters can lose electrons in the}

gas phase. Spectral features in an ATD, including tails, and even peaks, should not always be assigned to different structural families. It was shown that isomerization occurring on timescales smaller than or similar to that of the separation can yield characteristic signatures..$^{35,40,41}$ In this context, it is striking 
that the long tailing peaks to the short drift time side observed for $\left[\mathrm{Au}_{25}\right.$ ortho$\left.\mathrm{MBA}_{18}\right]^{-4}$, $\left[\mathrm{Au}_{25} \text { meta- } \mathrm{MBA}_{18}\right]^{-4}$, and $\left[\mathrm{Au}_{25} \text { para-MBA}{ }_{18}\right]^{-5}$ extend towards arrival times corresponding to that of $\left[\mathrm{Au}_{25} \mathrm{Ortho}-\mathrm{MBA}_{18}\right]^{-5},\left[\mathrm{Au}_{25} \text { meta-MBA}{ }_{18}\right]^{-5}$, and $\left[\mathrm{Au}_{25}\right.$ para$\left.\mathrm{MBA}_{18}\right]^{-6}$, respectively. Such coincidence would be consistent with a transformation occurring during the drift. Namely, ions starting their drift as $\left[\mathrm{Au}_{25} \text { meta-MBA } \mathrm{M}_{18}\right]^{-5}$ and losing an electron would be detected as $\left[\mathrm{Au}_{25} \text { meta-MBA} \mathrm{A}_{18}\right]^{-4}$. However, their

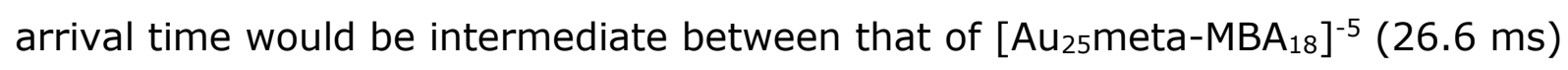
and $\left.\left[\mathrm{Au}_{25} \text { meta-MBA}\right]_{18}\right]^{-4}(32.6 \mathrm{~ms})$. This would result in the ATD of $\left[\mathrm{Au}_{25} \mathrm{meta}-\right.$ $\left.\mathrm{MBA}_{18}\right]^{-4}$ displaying a long tail, as that presently observed.

To test this hypothesis, we performed IMS-IMS-MS measurements by selecting $\left.\left[\mathrm{Au}_{25} \text { meta-MBA}\right]_{18}\right]^{-5}$ ions at the end of the first drift tube of our instrument. The ATDs recorded after selection for both $\left[\mathrm{Au}_{25} \text { meta- } M B A_{18}\right]^{-5}$ and $\left[\mathrm{Au}_{25} \text { meta-MBA}{ }_{18}\right]^{-}$ 4 , as shown in Figure 6 . The fact that significant signal is detected for [ $\mathrm{Au}_{25} \mathrm{meta}-$ $\left.\mathrm{MBA}_{18}\right]^{-4}$ when the selection window is centered on drift times characteristic of $\left.\left[\mathrm{Au}_{25} \text { meta-MBA}\right]_{18}\right]^{-5}$ clearly demonstrates that electron loss has occurred. The recorded ATD for $\left[\mathrm{Au}_{25} \text { meta-MBA }{ }_{18}\right]^{-4}$ are composed of a broad plateau. The plateau starts at the arrival time of the selected $\left[\mathrm{Au}_{25} \text { meta-MBA}{ }_{18}\right]^{-5}$ ions and extends to longer drift times. Ions that have lost an electron during their flight are responsible for this plateau. Those ions have been travelling part of their way through the tube as $\left[\mathrm{Au}_{25} \mathrm{meta}-\mathrm{MBA}_{18}\right]^{-5}$, and the other part as $\left[\mathrm{Au}_{25} \text { meta-MBA}{ }_{18}\right]^{-}$ 4 , after electron loss. As a consequence, their drift time is intermediate between

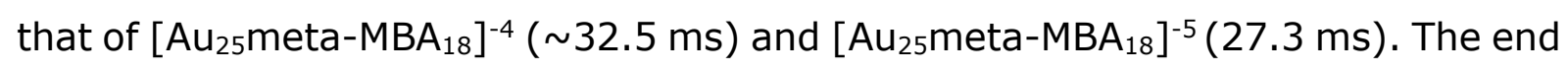
of the plateau corresponds to ions which have lost an electron immediately after selection and have then travelled all the way through DT2 as $\left[\mathrm{Au}_{25} \mathrm{meta}-\mathrm{MBA}_{18}\right]^{-4}$. Note that the apparent shift between the ends of the plateau in ATDs recorded with different selection windows is a simple consequence of the selection itself. As illustrated in Figure S6, the arrival times can be rescaled by subtracting the selection time in order to measure the drift time after selection. This way all plateaus end at the same time $(\sim 18.5 \mathrm{~ms})$, corresponding to the drift time of $\left[\mathrm{Au}_{25} \mathrm{meta}-\mathrm{MBA}_{18}\right]^{-4}$ through DT2 only.

Spontaneous electron loss in the gas phase was already observed for multiplycharged ions, ${ }^{42}$ and can be interpreted in terms of the Coulomb repulsion lowering the binding energy for an electron, potentially towards negative values. ${ }^{43}$ Tasaka et al. recently investigated electron photo-detachment in negatively charged $\mathrm{Ag}_{44}$ 
clusters with $\mathrm{SC}_{6} \mathrm{H}_{3} \mathrm{~F}_{2}$ ligands. ${ }^{44}$ They reported that such clusters were metastable yet at the 4- charge state, with a repulsive Coulomb barrier of $2.7 \mathrm{eV}$. We estimated the effect of Coulomb repulsion in our case, through a simple model for charges on a sphere. We considered $z$ charges distributed on a sphere of a radius compatible with that of the clusters (we estimated the radius $R$ from the average CCS of the clusters by $R=\sqrt{\frac{\langle C C S\rangle}{\pi}}$, yielding $\left.12 \AA\right)$. The optimal charge repartition was determined by numerically solving Thomson's problem for charges on a sphere, using the optimize.minimize routine from the Scipy library. ${ }^{36}$ Two charge distribution models were considered: all charges on the surface, or one charge at the center of the sphere. The Coulomb energy at each charged site was finally computed. We plotted the maximum value of these per-site energies as a function of $z$ in Figure 7. This simplistic model yields repulsive Coulomb energies comparable to the typical binding energy of the HOMO electron on a carboxylate $(\sim 3.5 \mathrm{eV})^{45}$, as soon as 5-6 charges are present. Although this model does not give any information on the energy barrier for electron detachment, it provides a rough estimate of the number of charges beyond which an electron may have a negative binding energy, thus being potentially able to spontaneously detach. Under this respect, the threshold around 5-6 charges is in good agreement with the interpretation of our experimental observations in terms of electron loss.

The ATDs in Figure 6.b have been normalized to the total ion signal (i.e. the sum of the intensities for $\left[\mathrm{Au}_{25} \mathrm{meta}_{\mathrm{MBA}} \mathrm{MB}^{-5}\right.$ and $\left[\mathrm{Au}_{25} \text { meta-MBA}{ }_{18}\right]^{-4}$ ions). This allows the intensities recorded after selection of the two IMS peaks ( $A$ and $B$ in Figure 6) to be compared. It clearly appears that the signal for $\left[\mathrm{Au}_{25} \text { meta-MBA} A_{18}\right]^{-4}$ is significantly higher after selection of the region $B$. More precisely, the ratio of the ion signal for $\left[\mathrm{Au}_{25} \text { meta- } \mathrm{MBA}_{18}\right]^{-4}$ to the total ion signal, which can be interpreted as a detachment yield is $13 \%$ and $27 \%$ after selection of $A$ and $B$ regions, respectively. If we consider the average drift time after selection, the corresponding lifetimes can be estimated to be $\tau_{A}=118 \mathrm{~ms}$ and $\tau_{B}=54 \mathrm{~ms}$. This difference further supports that the $\left[\mathrm{Au}_{25} \mathrm{meta}_{\mathrm{MBA}} \mathrm{MB}_{18}\right]^{-5}$ ion populations separated by IMS correspond to different structures, and possibly different charge repartition patterns. Based on the energetics of Coulomb repulsion included in the above model, the population in the A region, with a more compact structure, is also expected to display the lowest electron binding energy. We nevertheless observe slower electron loss from this population. This suggests that the detachment 
mechanisms for populations A and B may involve different electronic states, and possibly electrons localized in different regions of the cluster. Indeed, the extra charge for population $B$ is $x=0$, while population $A$ is dominated by $x=-1$. On the other hand, the dynamics for electron loss may also be limited by other factors, such as the existence of intermediate states involving a geometrical deformation of the cluster or the ligands before detachment. This would also be consistent with the timescales involved. Moreover, the tail on the long arrival times side on the ATDs for $\left[\mathrm{Au}_{25} \text { meta-MBA} 18\right]^{-5}$ ions (Figure 6.a) would be consistent with the latter hypothesis.

Consequences of electron loss on the observed charging patterns. The existence of electron detachment provides new insight in the above-described heterogeneities in the charging pattern of the clusters. After a cluster has lost an electron, its charge shift is increased by 1 . As illustrated in Scheme 1, this implies that part of the population with a charge shift $x=0$ at charge state $z$ may originate from clusters initially bearing $z+1$ negative charges (with a charge shift of -1 ), which have lost an electron before being detected. As a consequence, the population detected with an extra charge $x=0$ is a mixture of clusters natively displaying a 0 charge shift, and clusters natively displaying a -1 charge shift and having lost an electron.

Clusters with the same charge shift may then be detected at different arrival times (see Figure 5 and Figure S5) depending on whether they have lost an electron or not. Beyond the kinetic effects discussed in the above section, different arrival times generally correspond to different CCS. It is indeed likely that electron loss results in a structural change (then a change in CCS). A higher net charge generally results in a higher CCS (Figure S4). As already mentioned in the above section, electron loss may then be accompanied by partial structural re-organization of the cluster, especially of the ligands, due to the release of Coulombic repulsion. However, the timescale necessary for such structural relaxation is difficult to estimate, and long-lived metastable structural intermediates may be involved. On the other hand, even if complete structural relaxation occurs after electron loss, charges are most probably located on different sites for different populations of clusters. This also results in different CCSs.

A detailed interpretation of the complex processes that may be triggered by the loss of an electron nevertheless lies out of the scope of the present paper. Let us 
rather focus on the consequence of electron loss on the measured charge shift distributions summarized in Figure 2. In particular, the contribution of electron loss allows tentative interpretation of the observation that clusters with a -1 charge shift dominate for the lowest charge states, whereas clusters at higher charge states mainly display a 0 charge shift. The populations we observe are affected by the time-window of the detection. In other words, we see only species that survive long-enough to be detected. Our CID results (Figure 3 ) emphasized the decreased stability of clusters with -1 charge shift as compared to those that with $x=0$ or +1 . Moreover our toy model for Coulomb repulsion suggests that part of the charge located in the vicinity of the cluster core would result in an increase of the Coulomb repulsion at the peripheral charged sites (see Figure 7), and then favor electron detachment, even if other factors also influence electron loss kinetics. Clusters with a charge shift $x=-1$ may then not be stable enough to survive as highly charges species, even though such species are formed during the electrospray process.

Differences in lifetimes would then explain that species with $x=0$ (even $x=+1$, for ortho-MBA) dominate for the highest observed charge states. Under the present hypothesis, those clusters would mainly originate from metastable species with higher charge states, and a more negative charge shifts. Furthermore, the absence of higher charges states in the mass spectra (especially for clusters with orthoMBA) is consistent with this explanation, considering that those species are not stable during the timescale of the experiment. Importantly, as illustrated by our spherical model in Figure 7, the decrease of the Coulomb repulsion energy after electron loss (about $0.8 \mathrm{eV}$ per electron) is expected to be important enough to significantly increase the lifetime of the resulting species. Therefore, no more than two electron loss events are expected to occur within the timescale of the experiment. It is thus likely that part of the heterogeneity in the extra charge of the clusters reflects that different charging patterns pre-exist in solution. This is potentially the case for the observed proportion (about $10 \%$ ) of clusters with $x=0$ for the lowest charge states of meta- and para-MBA containing clusters. Moreover, the presence of clusters with $x=-2$, for clusters with ortho-MBA at charge state 3 cannot result from an artifact due to electron loss. 


\section{Conclusions}

Mass spectrometry and ion mobility spectrometry were used to investigate the heterogeneity in the charging patterns of $\mathrm{Au}_{25} \mathrm{MBA}_{18}$ clusters. We showed that for a given charge state of those clusters, several populations with different protonation patterns co-exist. Those populations display different stabilities with respect to collisional activation. Ion mobility experiments also demonstrate that they have different cross sections, and then display not only different charge locations, but also possibly different structures. However, no systematic correlation was found between the amount of charges not attributable to deprotonation and the CCS. In particular, the present results do not allow to emphasize potential charge-driven deformations of the cluster core. From the deconvolution of the ion mobility distributions, and based on tandem-ion mobility measurements, we gave evidence that part of the heterogeneity in the charging patterns of the clusters was attributable to the existence of spontaneous electron loss during the measurement. This effect has to be taken into account to avoid biases in data interpretation, especially when considering the highest charge states that are detected. Heterogeneities in structure and charging patterns are nevertheless present even for charge states that are not likely affected by the above-cited bias. This suggests that such heterogeneity pre-exists in solution, possibly in relation with different oxidation states of the cluster. Numerous wet chemistry strategies have been proposed to design atomically precise metal clusters, with remarkable monodispersity in terms of composition. The present work provides further evidence that other sources of dispersity have to be considered, which may significantly affect the physical and chemical properties of the clusters.

Supporting information: Scheme of the experimental setup, deconvoluted mass spectra for all investigated species, collision-induced fragmentation results, experimental CCS values, arrival-time-specific mass spectra for $\left[\mathrm{Au}_{25}\right.$ ortho$\left.\mathrm{MBA}_{18}\right]^{-5}$ and $\left[\mathrm{Au}_{25} \text { para-MBA}{ }_{18}\right]^{-6}$, rescaled IMS-IMS ATDs. 


\section{References}

(1) Jin, R. Quantum Sized, Thiolate-Protected Gold Nanoclusters. Nanoscale 2010, 2 (3), 343-362. https://doi.org/10.1039/B9NR00160C.

(2) Zeng, C.; Chen, Y.; Das, A.; Jin, R. Transformation Chemistry of Gold Nanoclusters: From One Stable Size to Another. J. Phys. Chem. Lett. 2015, 6 (15), 2976-2986. https://doi.org/10.1021/acs.jpclett.5b01150.

(3) Jin, R.; Zeng, C.; Zhou, M.; Chen, Y. Atomically Precise Colloidal Metal Nanoclusters and Nanoparticles: Fundamentals and Opportunities. Chem. Rev. 2016, 116 (18), 10346-10413. https://doi.org/10.1021/acs.chemrev.5b00703.

(4) Chakraborty, I.; Pradeep, T. Atomically Precise Clusters of Noble Metals: Emerging Link between Atoms and Nanoparticles. Chem. Rev. 2017, 117 (12), 8208-8271. https://doi.org/10.1021/acs.chemrev.6b00769.

(5) Kang, X.; Chong, H.; Zhu, M. $\operatorname{Au}_{25}(\mathrm{SR})_{18}$ : The Captain of the Great Nanocluster Ship. Nanoscale 2018, 10 (23), 10758-10834. https://doi.org/10.1039/C8NR02973C.

(6) Zhu, M.; Aikens, C. M.; Hollander, F. J.; Schatz, G. C.; Jin, R. Correlating the Crystal Structure of A Thiol-Protected $\mathrm{Au}_{25}$ Cluster and Optical Properties. J. Am. Chem. Soc. 2008, 130 (18), 5883-5885. https://doi.org/10.1021/ja801173r.

(7) Heaven, M. W.; Dass, A.; White, P. S.; Holt, K. M.; Murray, R. W. Crystal Structure of the Gold Nanoparticle $\left[\mathrm{N}\left(\mathrm{C}_{8} \mathrm{H}_{17}\right)_{4}\right]\left[\mathrm{Au}_{25}\left(\mathrm{SCH}_{2} \mathrm{CH}_{2} \mathrm{Ph}\right)_{18}\right]$. J. Am. Chem. Soc. 2008, 130 (12), 3754-3755. https://doi.org/10.1021/ja800561b.

(8) Negishi, Y.; Chaki, N. K.; Shichibu, Y.; Whetten, R. L.; Tsukuda, T. Origin of Magic Stability of Thiolated Gold Clusters: A Case Study on $\mathrm{Au}_{25}\left(\mathrm{SC}_{6} \mathrm{H}_{13}\right)_{18}$. J. Am. Chem. Soc. 2007, 129 (37), 11322-11323. https://doi.org/10.1021/ja073580+.

(9) Zhu, M.; Eckenhoff, W. T.; Pintauer, T.; Jin, R. Conversion of Anionic [Au25(SCH2CH2 Ph)18 ]- Cluster to Charge Neutral Cluster via Air Oxidation. J. Phys. Chem. C 2008, 112 (37), 14221-14224. 
https://doi.org/10.1021/jp805786p.

(10) Tofanelli, M. A.; Salorinne, K.; Ni, T. W.; Malola, S.; Newell, B.; Phillips, B.; Häkkinen, H.; Ackerson, C. J. Jahn-Teller Effects in $\mathrm{Au}_{25}(\mathrm{SR})_{18}$. Chem. Sci. 2016, 7 (3), 1882-1890. https://doi.org/10.1039/C5SC02134K.

(11) Zhu, M.; Aikens, C. M.; Hendrich, M. P.; Gupta, R.; Qian, H.; Schatz, G. C.; Jin, R. Reversible Switching of Magnetism in Thiolate-Protected Au 25 Superatoms. J. Am. Chem. Soc. 2009, 131 (7), 2490-2492. https://doi.org/10.1021/ja809157f.

(12) Chong, H.; Li, P.; Wang, S.; Fu, F.; Xiang, J.; Zhu, M.; Li, Y. Au 25 Clusters as Electron-Transfer Catalysts Induced the Intramolecular Cascade Reaction of 2-Nitrobenzonitrile. Sci. Rep. 2013, 3 (1), 3214. https://doi.org/10.1038/srep03214.

(13) Antonello, S.; Dainese, T.; Maran, F. Exploring Collective Substituent Effects: Dependence of the Lifetime of Charged States of $\mathrm{Au}_{25}\left(\mathrm{SC}_{n} \mathrm{H}_{2 n+1}\right)_{18}$ Nanoclusters on the Length of the Thiolate Ligands. Electroanalysis 2016, 28 (11), 2771-2776. https://doi.org/10.1002/elan.201600323.

(14) Collins, C. B.; Tofanelli, M. A.; Crook, M. F.; Phillips, B. D.; Ackerson, C. J. Practical Stability of $\mathrm{Au}_{25}(\mathrm{SR})_{18}-1 / 0 /+1 . R S C A d v .2017,7$ (71), 4506145065. https://doi.org/10.1039/C7RA07511A.

(15) Antonello, S.; Perera, N. V.; Ruzzi, M.; Gascón, J. A.; Maran, F. Interplay of Charge State, Lability, and Magnetism in the Molecule-like $\mathrm{Au}_{25}(\mathrm{SR})_{18}$ Cluster. J. Am. Chem. Soc. 2013, 135 (41), 15585-15594. https://doi.org/10.1021/ja407887d.

(16) Hamouda, R.; Bellina, B.; Bertorelle, F.; Compagnon, I.; Antoine, R.; Broyer, M.; Rayane, D.; Dugourd, P. Electron Emission of Gas-Phase $\left[\mathrm{Au}_{25}(\mathrm{SG})_{18^{-}}\right.$ $6 \mathrm{H}^{7-}$ Gold Cluster and Its Action Spectroscopy. J. Phys. Chem. Lett. 2010, 1 (21), 3189-3194. https://doi.org/doi: 10.1021/jz101287m.

(17) Negishi, Y.; Takasugi, Y.; Sato, S.; Yao, H.; Kimura, K.; Tsukuda, T. MagicNumbered $A u_{n}$ Clusters Protected by Glutathione Monolayers ( $\mathrm{n}=18,21$, 25, 28, 32, 39): Isolation and Spectroscopic Characterization. J. Am. Chem. Soc. 2004, 126 (21), 6518-6519. https://doi.org/10.1021/ja0483589. 
(18) Negishi, Y.; Nobusada, K.; Tsukuda, T. Glutathione-Protected Gold Clusters Revisited: Bridging the Gap between Gold(I)-Thiolate Complexes and Thiolate-Protected Gold Nanocrystals. J. Am. Chem. Soc. 2005, 127 (14), 5261-5270. https://doi.org/10.1021/ja042218h.

(19) Hamouda, R.; Bertorelle, F.; Rayane, D.; Antoine, R.; Broyer, M.; Dugourd, P. Glutathione Capped Gold $\mathrm{Au}_{\mathrm{N}}(\mathrm{SG})_{\mathrm{M}}$ Clusters Studied by Isotope-Resolved Mass Spectrometry. Int. J. Mass Spectrom. 2013, 335, 1-6. https://doi.org/10.1016/j.ijms.2012.10.008.

(20) Bertorelle, F.; Russier-Antoine, I.; Comby-Zerbino, C.; Chirot, F.; Dugourd, P.; Brevet, P.-F.; Antoine, R. Isomeric Effect of Mercaptobenzoic Acids on the Synthesis, Stability, and Optical Properties of $\mathrm{Au}_{25}(\mathrm{MBA})_{18}$ Nanoclusters. ACS Omega 2018, 3 (11), 15635-15642. https://doi.org/10.1021/acsomega.8b02615.

(21) Juarez-Mosqueda, R.; Mpourmpakis, G. Elucidating the Optical Spectra of $\left[\mathrm{Au}_{25}(\mathrm{SR})_{18}\right]^{\mathrm{q}}$ Nanoclusters. Phys. Chem. Chem. Phys. 2019, 21 (40), 22272-22282. https://doi.org/10.1039/C9CP03982A.

(22) Hirata, K.; Tomihara, R.; Kim, K.; Koyasu, K.; Tsukuda, T. Characterization of Chemically Modified Gold and Silver Clusters in Gas Phase. Phys. Chem. Chem. 2019, 21 (32), 17463-17474. https://doi.org/10.1039/C9CP02622C.

(23) Baksi, A.; Ghosh, A.; Mudedla, S. K.; Chakraborty, P.; Bhat, S.; Mondal, B.; Krishnadas, K. R.; Subramanian, V.; Pradeep, T. Isomerism in Monolayer Protected Silver Cluster Ions: An Ion Mobility-Mass Spectrometry Approach. J. Phys. Chem. C 2017, 121 (24), 13421-13427. https://doi.org/10.1021/acs.jpcc.7b04559.

(24) Comby-Zerbino, C.; Bertorelle, F.; Chirot, F.; Dugourd, P.; Antoine, R. Structural Insights into Glutathione-Protected Gold $\operatorname{Au}_{10-12}(\mathrm{SG})_{10-12}$ Nanoclusters Revealed by Ion Mobility Mass Spectrometry. Eur. Phys. J. D 2018, 72 (8), 144. https://doi.org/10.1140/epjd/e2018-90133-8.

(25) Harkness, K. M.; Fenn, L. S.; Cliffel, D. E.; Mclean, J. A. Surface Fragmentation of Complexes from Thiolate Protected Gold Nanoparticles by Ion Mobility-Mass Spectrometry. Anal. Chem. 2010, 82 (7), 3061-3066. 
https://doi.org/10.1021/ac100251d.

(26) Soleilhac, A.; Bertorelle, F.; Comby-Zerbino, C.; Chirot, F.; Calin, N.; Dugourd, P.; Antoine, R. Size Characterization of Glutathione-Protected Gold Nanoclusters in the Solid, Liquid and Gas Phases. J. Phys. Chem. C 2017, 121 (49), 27733-27740. https://doi.org/10.1021/acs.jpcc.7b09500.

(27) Comby-Zerbino, C.; Perić, M.; Bertorelle, F.; Chirot, F.; Dugourd, P.; Bonačić-Koutecký, V.; Antoine, R. Catenane Structures of Homoleptic Thioglycolic Acid-Protected Gold Nanoclusters Evidenced by Ion MobilityMass Spectrometry and DFT Calculations. Nanomaterials 2019, 9 (3), 457. https://doi.org/10.3390/nano9030457.

(28) Ligare, M. R.; Baker, E. S.; Laskin, J.; Johnson, G. E. Ligand Induced Structural Isomerism in Phosphine Coordinated Gold Clusters Revealed by Ion Mobility Mass Spectrometry. Chem. Commun. 2017, 53 (53), 73897392. https://doi.org/10.1039/C7CC02251D.

(29) Simon, A.-L.; Chirot, F.; Choi, C. M.; Clavier, C.; Barbaire, M.; Maurelli, J.; Dagany, X.; MacAleese, L.; Dugourd, P. Tandem Ion Mobility Spectrometry Coupled to Laser Excitation. Rev. Sci. Instrum. 2015, 86 (9), 094101. https://doi.org/10.1063/1.4930604.

(30) Revercomb, H. E.; Mason, E. A. Theory of Plasma Chromatography, Gazeous Electrophoresis - Review. Anal. Chem. 1975, 47 (7), 970-983. https://doi.org/10.1021/ac60357a043.

(31) Choi, C. M.; MacAleese, L.; Dugourd, P.; Choi, M. C.; Chirot, F. PhotoInduced Linkage Isomerization in the Gas Phase Probed by Tandem Ion Mobility and Laser Spectroscopy. Phys. Chem. Chem. Phys. 2018, 20 (17), 12223-12228. https://doi.org/10.1039/C8CP01833B.

(32) Choi, C. M.; Simon, A.-L.; Chirot, F.; Kulesza, A.; Knight, G.; Daly, S.; MacAleese, L.; Antoine, R.; Dugourd, P. Charge, Color, and Conformation: Spectroscopy on Isomer-Selected Peptide Ions. J. Phys. Chem. B 2016, 120 (4), 709-714. https://doi.org/10.1021/acs.jpcb.5b11919.

(33) Choi, C. M.; Kulesza, A.; Daly, S.; MacAleese, L.; Antoine, R.; Dugourd, P.; Chirot, F. Ion Mobility Resolved Photo- fragmentation to Discriminate Protomers. Rapid Commun. Mass Spectrom. 2019, 33 (S1), 28-34. 
https://doi.org/10.1002/rcm.8202.

(34) Koeniger, S. L.; Clemmer, D. E. Resolution and Structural Transitions of Elongated States of Ubiquitin. J. Am. Soc. Mass Spectrom. 2007, 18 (2), 322-331. https://doi.org/10.1016/j.jasms.2006.09.025.

(35) Poyer, S.; Comby-Zerbino, C.; Choi, C. M.; MacAleese, L.; Deo, C.; Bogliotti, N.; Xie, J.; Salpin, J.-Y.; Dugourd, P.; Chirot, F. Conformational Dynamics In Ion Mobility Data. Anal. Chem. 2017, 89 (7), 4230-4237. https://doi.org/10.1021/acs.analchem.7b00281.

(36) Virtanen, P.; Gommers, R.; Oliphant, T. E.; Haberland, M.; Reddy, T.; Cournapeau, D.; Burovski, E.; Peterson, P.; Weckesser, W.; Bright, J.; et al. SciPy 1.0: Fundamental Algorithms for Scientific Computing in Python. Nat. Methods 2020, 17 (3), 261-272. https://doi.org/10.1038/s41592-0190686-2.

(37) Gohlke, C. molmass 2020.1.1 https://pypi.org/project/molmass/ (accessed May 31, 2020).

(38) Lugo, G.; Schwanen, V.; Fresch, B.; Remacle, F. Charge Redistribution Effects on the UV-Vis Spectra of Small Ligated Gold Clusters: A Computational Study. J. Phys. Chem. C 2015, 119 (20), 10969-10980. https://doi.org/10.1021/jp511120j.

(39) Wing-Bocanegra, A.; Tlahuice-Flores, A. Effect of the Charge State on Bare Monoicosahedral $\left[\mathrm{Au}_{13}\right]^{\mathrm{Z}+}$ and Diphosphine-Protected $\mathrm{Au}_{13}$ Clusters $\left[\mathrm{Au}_{13}(\mathrm{Dmpe})_{5} \mathrm{Cl}_{2}\right]^{\mathrm{Z}+}$ : Structural, Electronic and Vibrational DFT Studies. Phys. Chem. Chem. Phys. 2019, 21 (43), 23855-23864. https://doi.org/10.1039/C9CP04827H.

(40) Wyttenbach, T.; Pierson, N. A.; Clemmer, D. E.; Bowers, M. T. Ion Mobility Analysis of Molecular Dynamics. Annu. Rev. Phys. Chem. 2014, 65 (1), 175196. https://doi.org/10.1146/annurev-physchem-040513-103644.

(41) Kinnear, B. S.; Hartings, M. R.; Jarrold, M. F. Helix Unfolding in Unsolvated Peptides. J. Am. Chem. Soc. 2001, 123 (24), 5660-5667. https://doi.org/10.1021/ja004196e.

(42) Wang, X.-B.; Wang, L.-S. Observation of Negative Electron-Binding Energy 
in a Molecule. Nature 1999, 400 (6741), 245-248. https://doi.org/10.1038/22286.

(43) Wang, L.-S.; Wang, X.-B. Probing Free Multiply Charged Anions Using Photodetachment Photoelectron Spectroscopy. J. Phys. Chem. A 2000, 104 (10), 1978-1990. https://doi.org/10.1021/jp9940093.

(44) Tasaka, Y.; Nakamura, K.; Malola, S.; Hirata, K.; Kim, K.; Koyasu, K.; Häkkinen, H.; Tsukuda, T. Electron Binding in a Superatom with a Repulsive Coulomb Barrier: The Case of $\left[\mathrm{Ag}_{44}\left(\mathrm{SC}_{6} \mathrm{H}_{3} \mathrm{~F}_{2}\right)_{30}\right]^{4-}$ in the Gas Phase. J. Phys. Chem. Lett. 2020, 11 (8), 3069-3074. https://doi.org/10.1021/acs.jpclett.0c00786.

(45) Antoine, R.; Joly, L.; Allouche, A. R.; Broyer, M.; Lemoine, J.; Dugourd, P. Electron Photodetachment of Trapped Doubly Deprotonated Angiotensin Peptides. UV Spectroscopy and Radical Recombination. Eur. Phys. J. D 2009, 51 (1), 117-124. https://doi.org/10.1140/epjd/e2008-00076-4. 


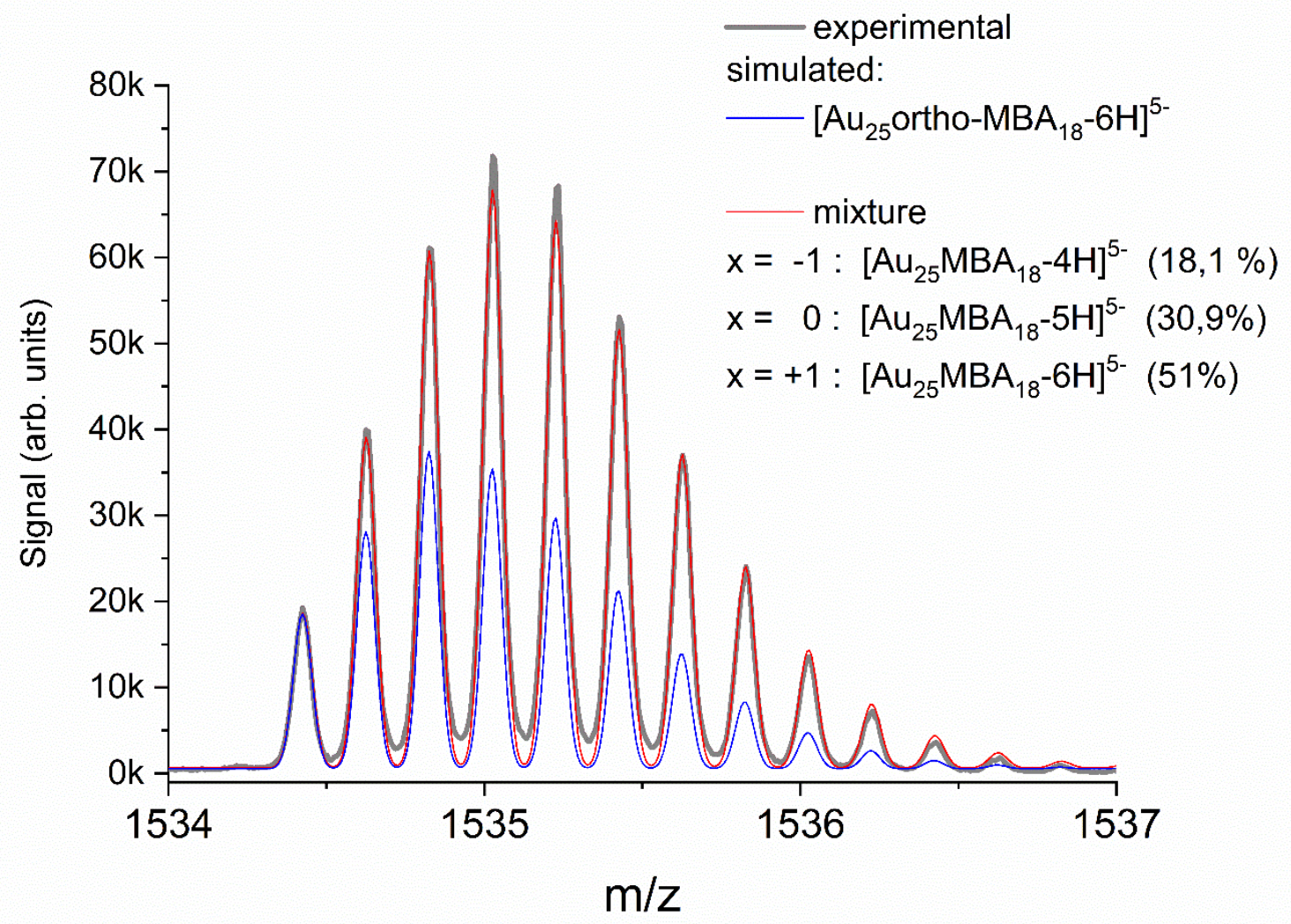

Figure 1: (black line) experimental isotope pattern for $\left[\mathrm{Au}_{25} \text { ortho- } \mathrm{MBA}_{18}\right]^{-5}$. The blue line corresponds to the simulated isotope patters for $\left[\mathrm{Au}_{25} \mathrm{Or} \text { tho-MBA } \mathrm{MB}_{18}-6 \mathrm{H}\right]^{-5}$ adjusted to fit the intensity of the experimental monoisotopic peak. The red line is a fit obtained by considering a mixture of species with different de-protonation patterns, corresponding to different charge shifts $x$. 

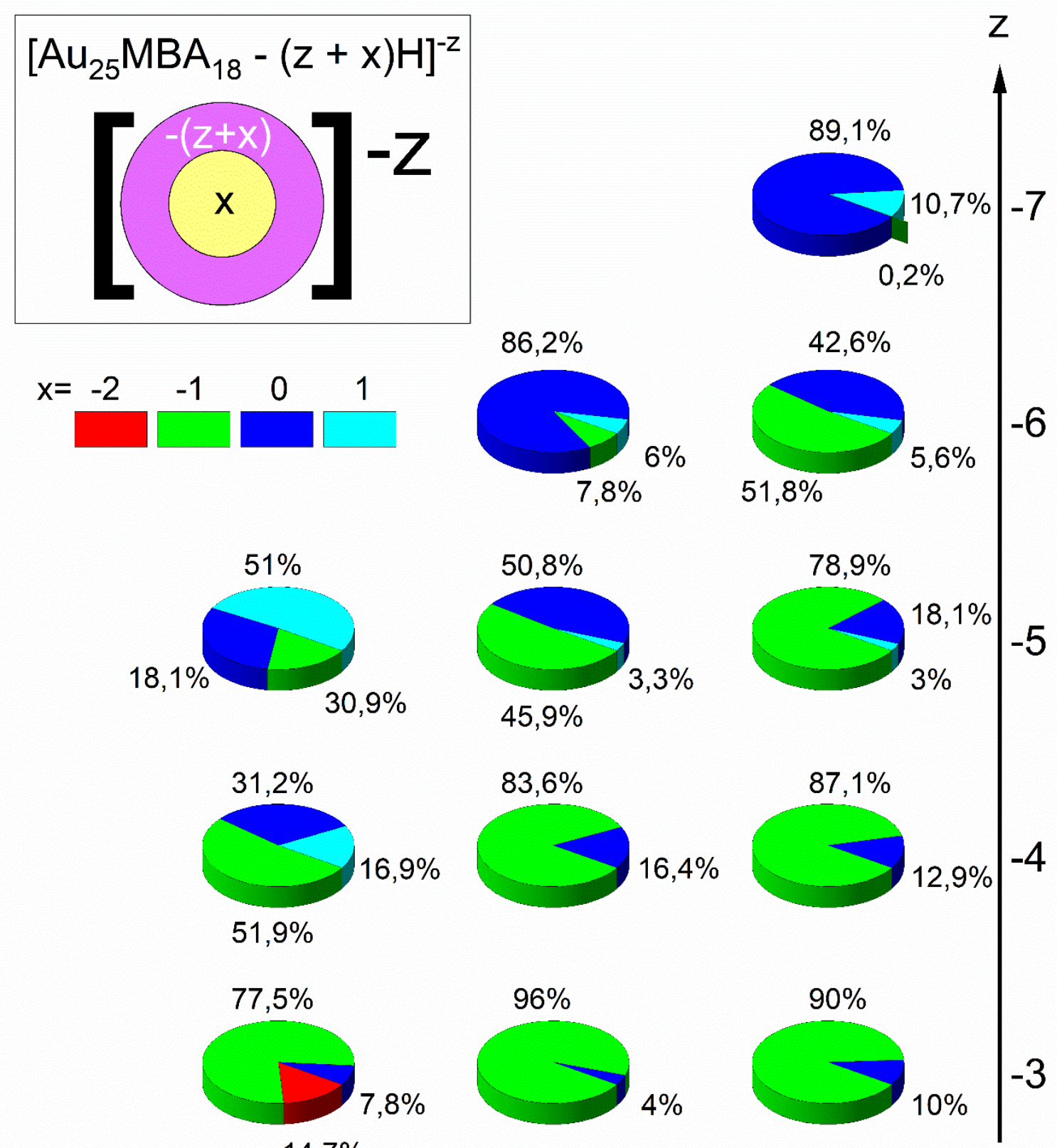

$14,7 \%$

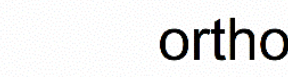

meta

para

ligand

Figure 2: The inset illustrates the charge repartition corresponding to a charge shift $x$ for a cluster with total charge $-z$ : $z+x$ negative charges are borne by deprotonated ligand carboxyl groups, and the remaining $x$ charges are located elsewhere inside the cluster. $x$ can be positive or negative. The pie charts represent the relative proportions of populations exhibiting different charge 
repartitions, characterized by different values of $x$, as deduced from deconvolution of the experimental mass spectra.

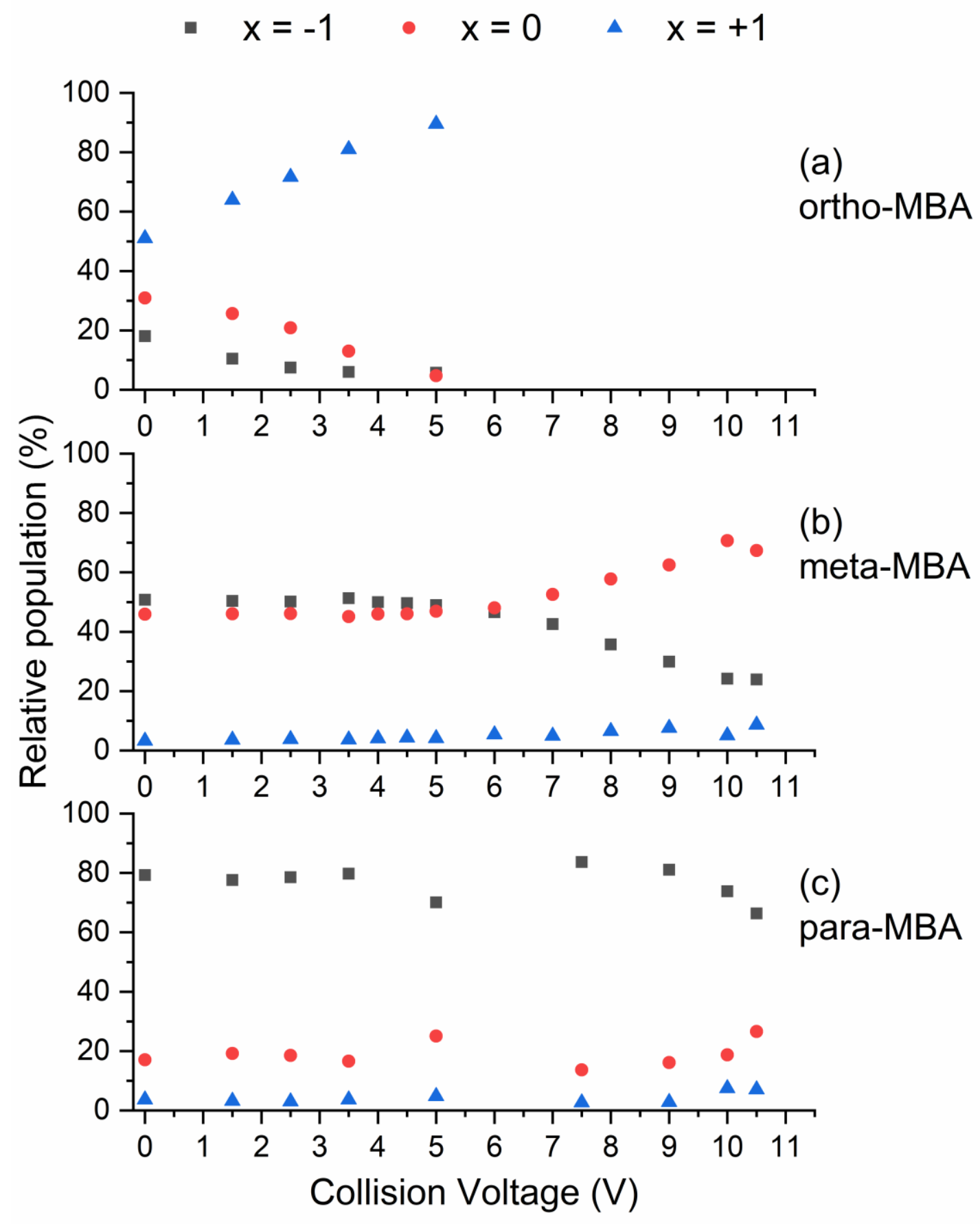

Figure 3: Evolution of the relative population of clusters with different charge shifts $\mathrm{x}$ in the precursor ion as a function of the collision voltage. Populations corresponding to $\mathrm{x}=0,-1$ and +1 are represented by red circles, black squares, and blue triangles, respectively. The selected precursor ion was the peak at 
$\mathrm{m} / \mathrm{z}$ 1536, corresponding to the 5- charge state of the clusters. Panels (a), (b), and (c) display the results obtained with ortho-, meta-, and para-MBA ligands, respectively.
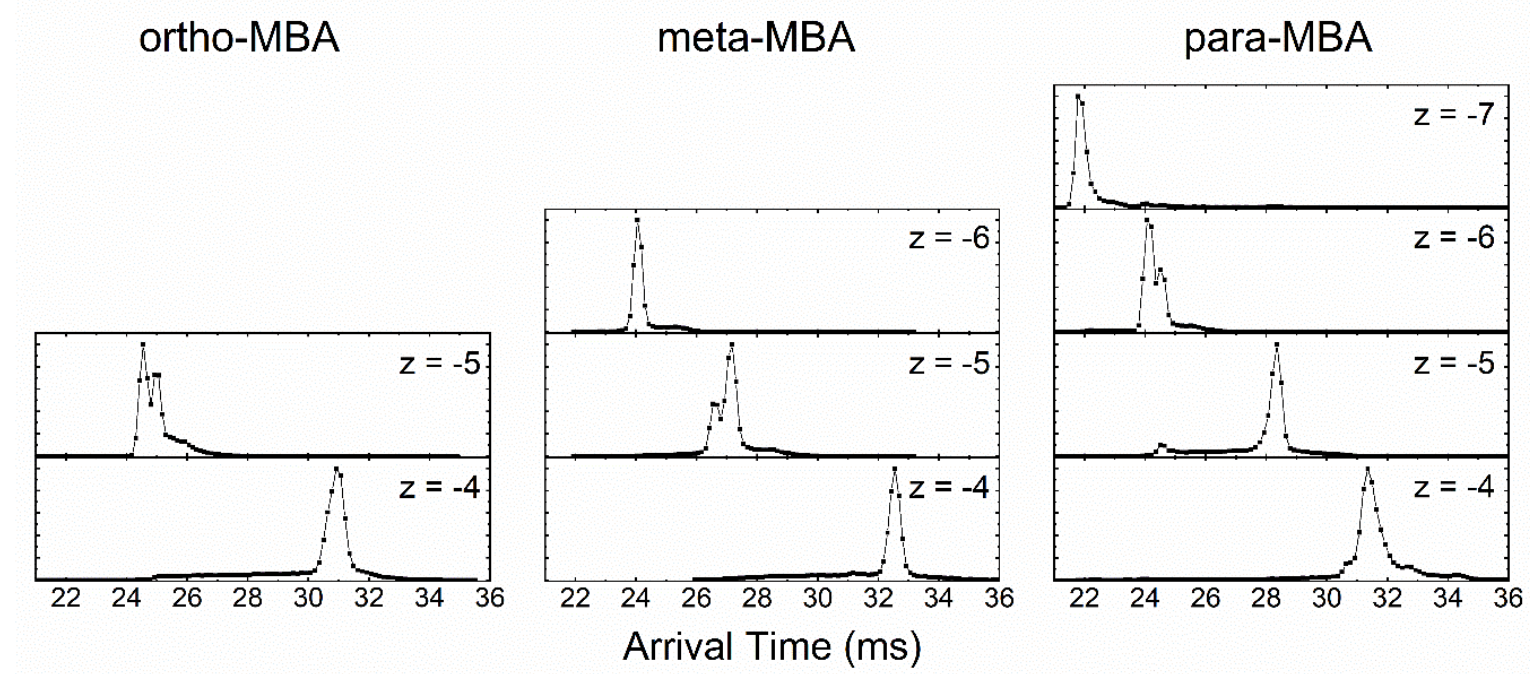

Figure 4: High-resolution arrival time distributions obtained in dual-drift tube configuration by integrating over the full isotope pattern for each charge state. Drift voltage $=1000 \mathrm{~V}$, drift length $=1.6 \mathrm{~m}$, drift gas: He, drift pressure: 4.0 Torr, drift temperature: 298K. 

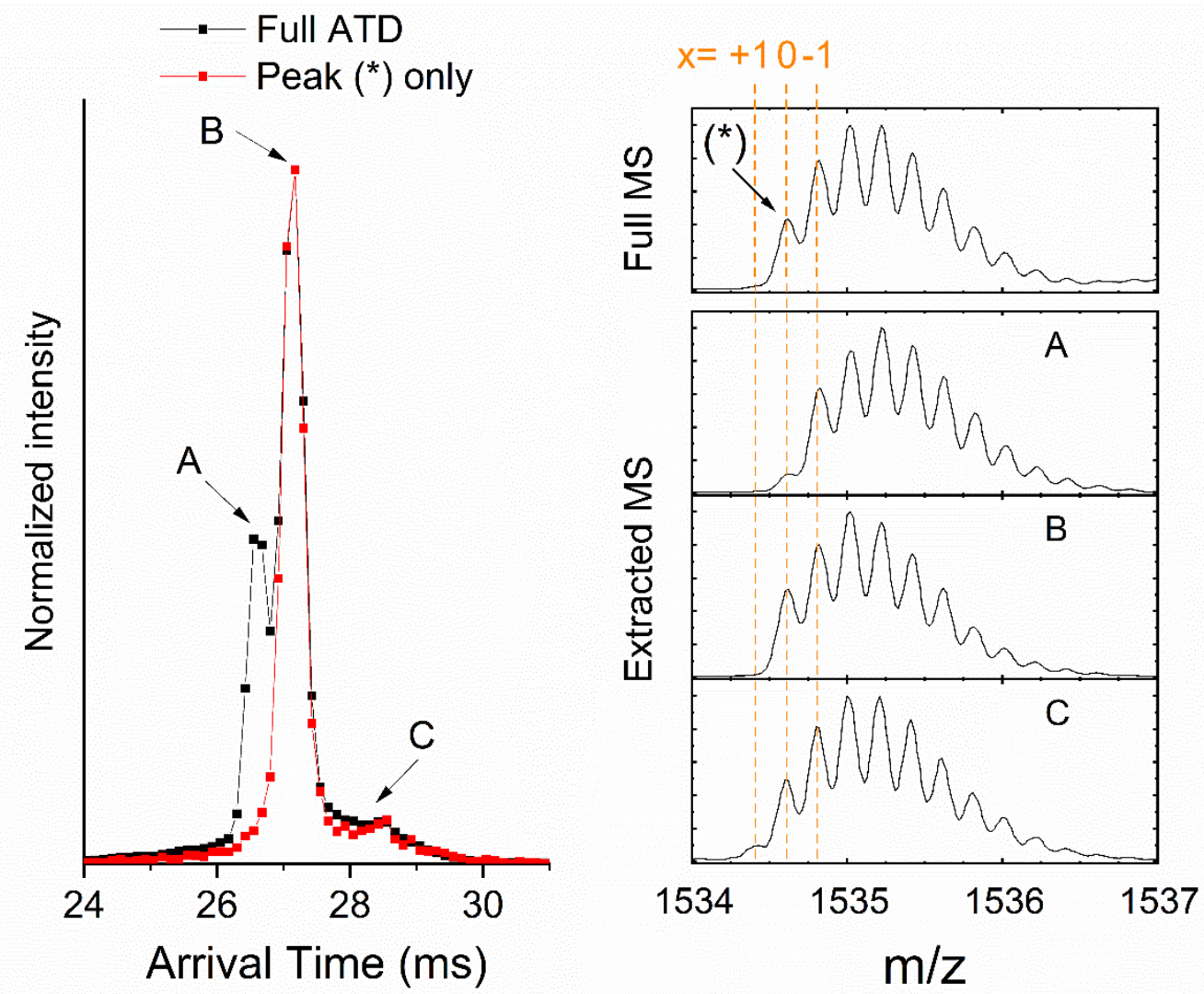

Figure 5: (Left) Extracted ATDs for $\left[\mathrm{Au}_{25} \mathrm{meta}-\mathrm{MBA}_{18}\right]^{-5}$ clusters from the full isotopic pattern (black), or (red) for the first isotopic peak visible at $\mathrm{m} / \mathrm{z} 1534.6$ $\left.{ }^{*}\right)$. (Right) Full mass spectrum and extracted mass spectra around $\mathrm{m} / \mathrm{z} 1535$. The extracted mass spectra are labelled $A, B$, and $C$ in relation with the selected arrival time extraction windows, corresponding to the different spectral features in the ATD. The dashed lines indicate the monoisotopic masses of different deprotonation patterns corresponding to different charge shifts $x$ ranging from -1 to +1 , and a total charge $z=-5$. 


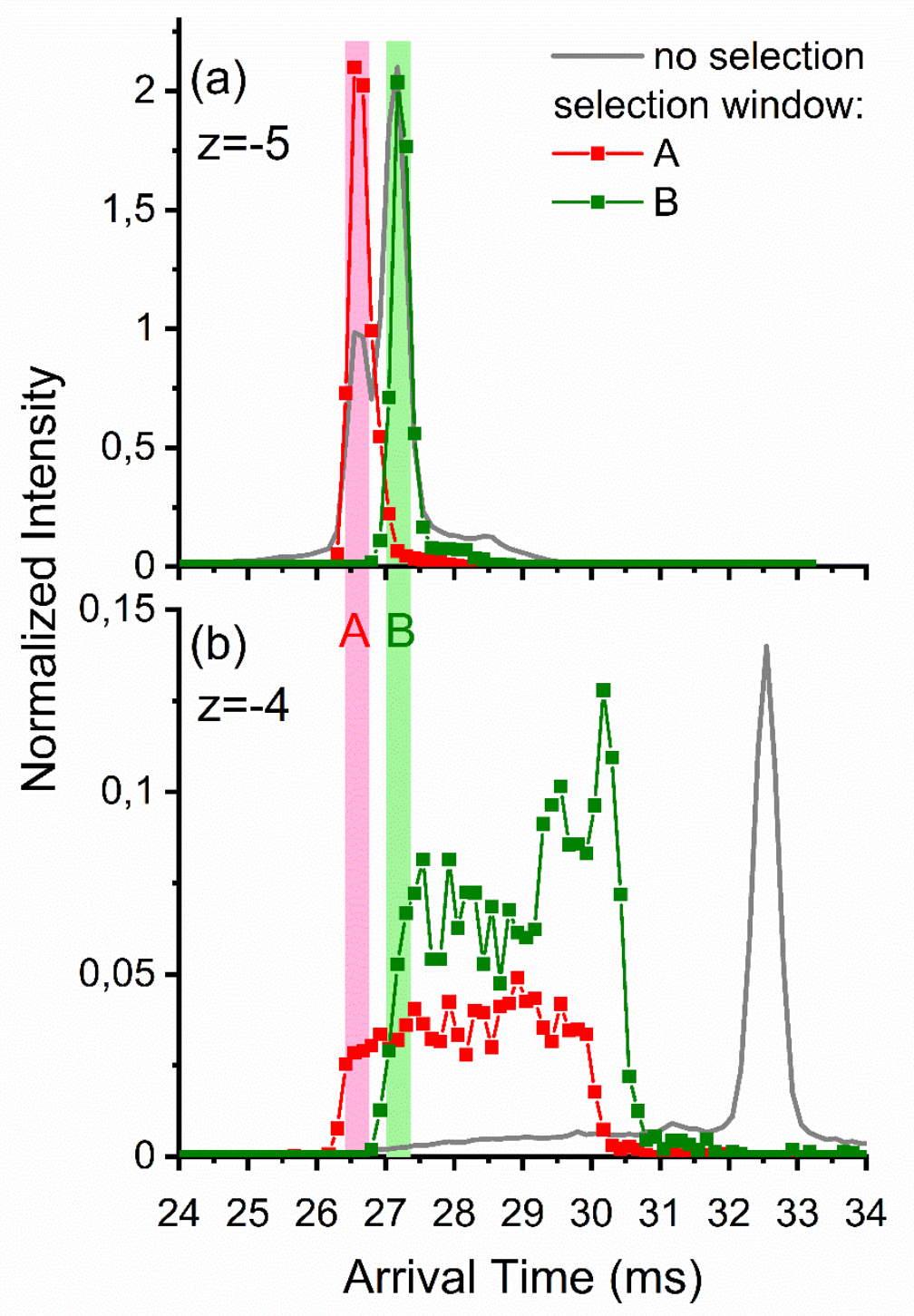

Figure 6: Arrival time distributions extracted for (a) $\left[\mathrm{Au}_{25} \text { meta-MBA}{ }_{18}\right]^{-5}$ clusters, and (b) $\left[\mathrm{Au}_{25} \text { meta-MBA }{ }_{18}\right]^{-4}$ clusters, by integrating over the full isotopic pattern in each case. Red and green traces were obtained after selection of the ions on the fly (between the two drift tubes), respectively using the selection windows labelled A and B. These ATDs were normalized to the total ion signal (see text). The corresponding ATDs obtained without selection are also provided as a reference (grey lines). 


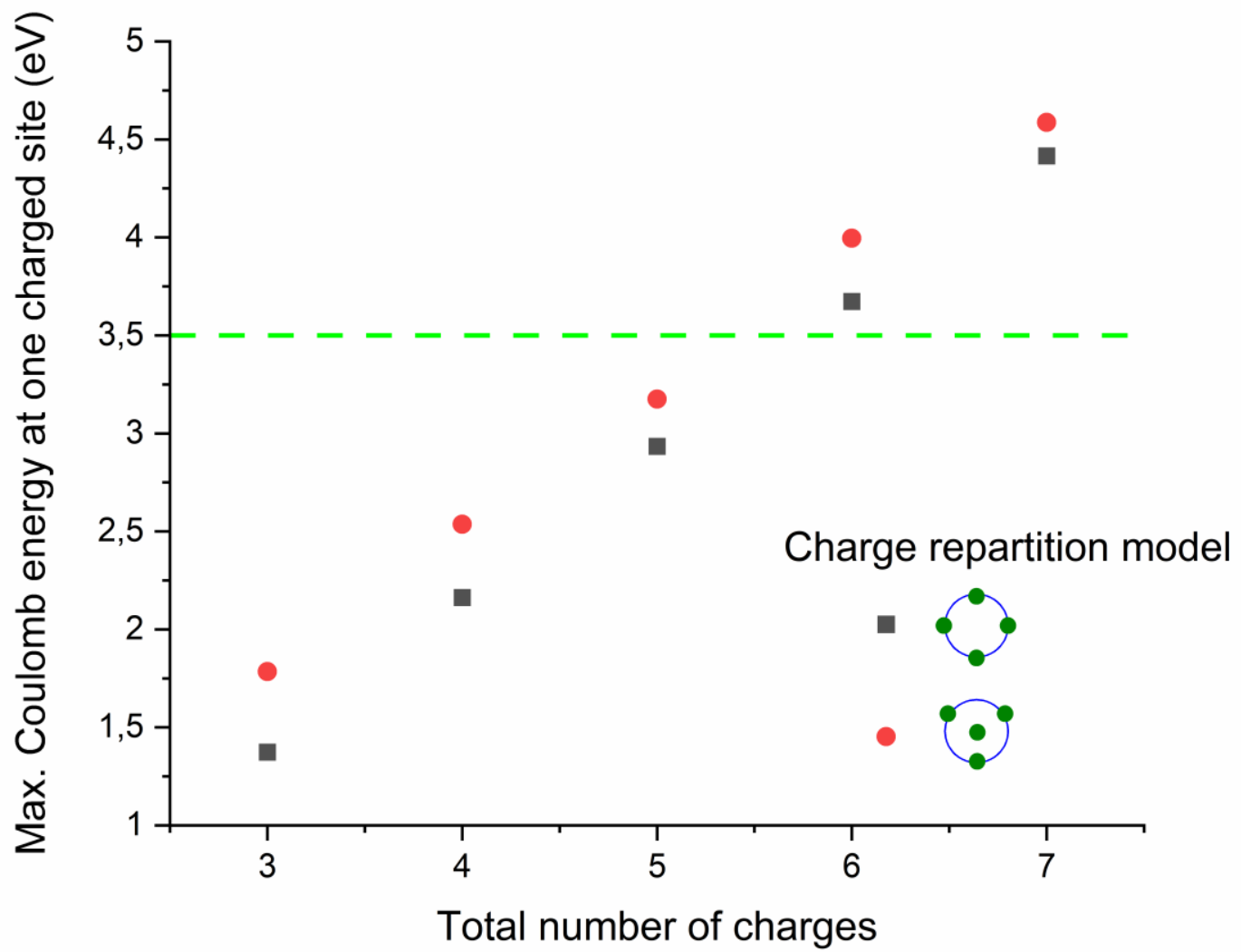

Figure 7: Coulomb repulsion energies calculated for charges constrained on a sphere with a radius comparable to that of the clusters. The reported values correspond to the maximum Coulomb energy at one charged site on the surface of the sphere. Two repartition models were considered: all charges on the surface of the sphere (black squares), or one charge at the center (red circles). The green dotted line represents a typical value for the binding energy of an electron on a carboxylate, taken from ref. 45. 


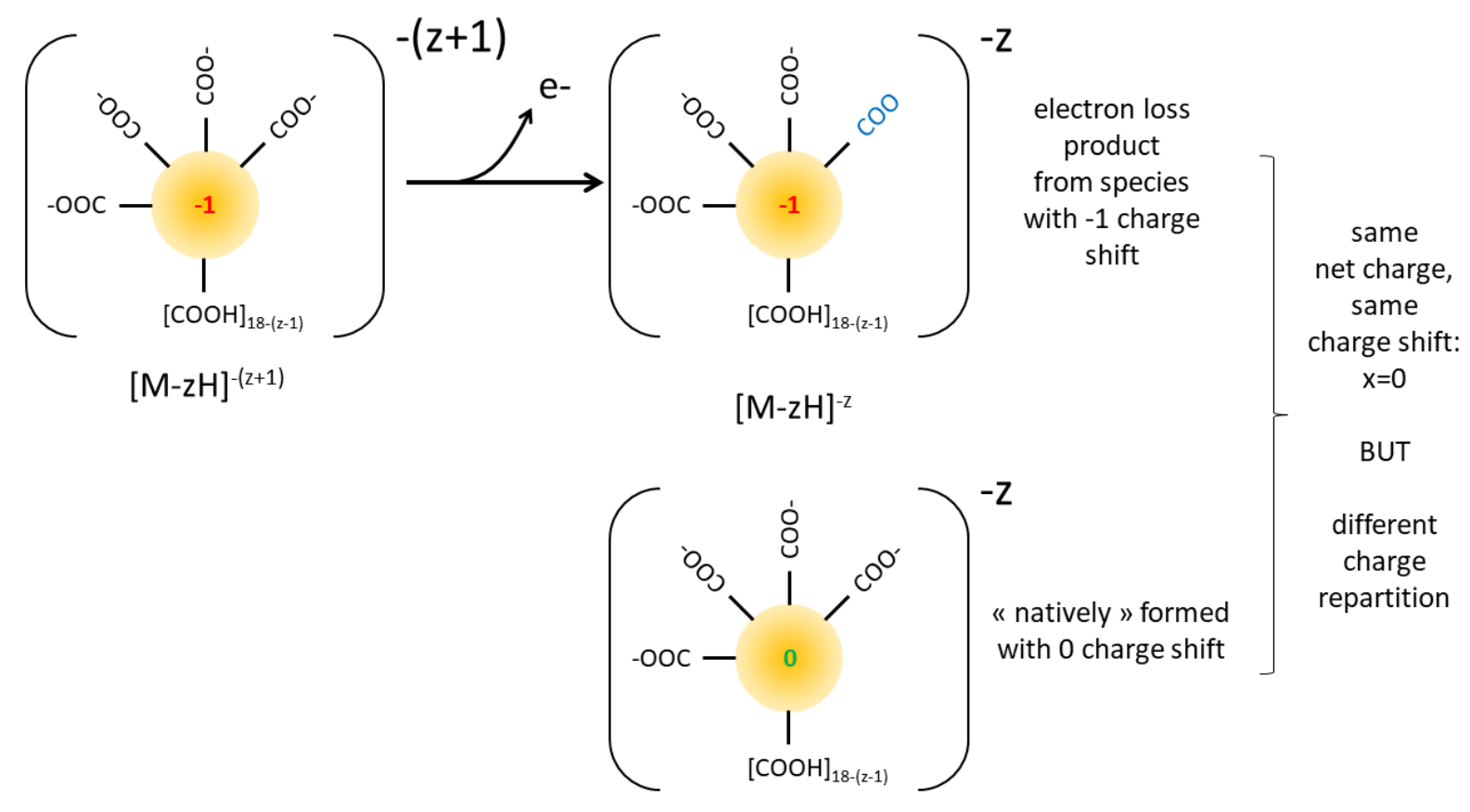

Scheme 1: Illustration of the impact of electron loss on the heterogeneity of clusters sharing the same apparent charging pattern. For simplicity, the charge shift was labelled at the center of the cluster. 


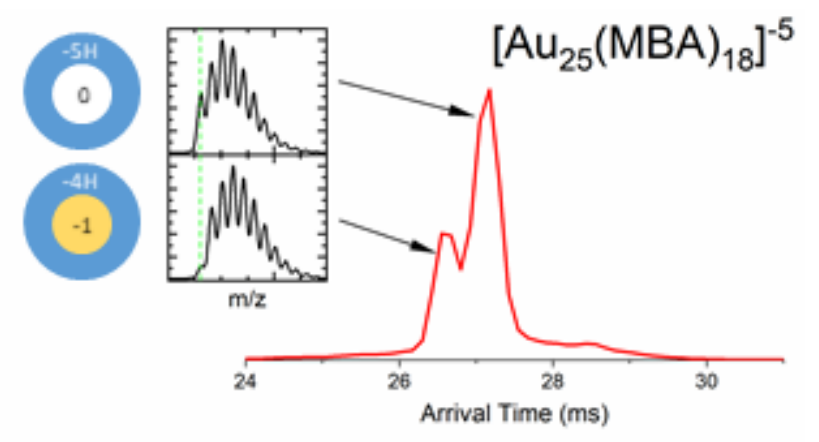

\section{TOC Graphics Structure and charge heterogeneity in isomeric $\mathrm{Au}_{25}(\mathrm{MBA})_{18}$ nanoclusters - insights from ion mobility and mass spectrometry}

Clothilde Comby-Zerbino, ${ }^{1}$ Franck Bertorelle, ${ }^{1}$ Philippe Dugourd, ${ }^{1}$ Rodolphe Antoine, ${ }^{* 1}$ and Fabien Chirot*2

${ }^{1}$ Univ Lyon, Université Claude Bernard Lyon 1, CNRS, UMR5306 Institut Lumière Matière, F-69100, 5 rue de la Doua, VILLEURBANNE, France

¿Univ Lyon, Université Claude Bernard Lyon 1, CNRS, UMR5280 Institut des Sciences Analytiques, F-69100, 5 rue de la Doua, VILLEURBANNE, France

*Contact: fabien.chirot@univ-lyon1.fr, rodolphe.antoine@univ-lyon1.fr

\section{Abstract:}

Atomically precise $\mathrm{Au}_{25}(\mathrm{MBA})_{18}$ nanoclusters were investigated by mass spectrometry and ion mobility spectrometry. We show that clusters sharing the same chemical composition and bearing the same net charge may display different structures and different charge repartition patterns, in relation with different deprotonation states of the ligands. Part of the observed heterogeneity is a consequence of spontaneous electron loss occurring in the gas phase, which modifies the net charge of the clusters, while maintaining the initial protonation state. 


\section{Introduction}

The physico-chemical properties of small metal nanoclusters, composed of less than a hundred atoms, dramatically depend on their composition and structure. Different wet chemistry synthesis strategies have been developed to precisely control those parameters at the atomic level, and then design species with tailored properties. Such strategies have been especially fruitful to obtain gold nanoclusters stabilized by thiolated ligands (SR), yielding species of general composition $\left(A u_{n} \mathrm{SR}_{m}\right){ }^{1-4}$ Among these nanoclusters, $\mathrm{Au}_{25} \mathrm{SR}_{18}$ has a special place due to its high stability and its ease of preparation and functionalization..$^{5-7}$ Depending on the conditions of the preparation and storage, various charge states of $A_{25} \mathrm{SR}_{18}$ have been figured out (e.g. $\left.\left[\mathrm{Au}_{25} \mathrm{SR}_{18}\right]^{-1,0+1}\right) .^{8-10} \mathrm{Au}_{25} \mathrm{SR}_{18}$ nanoclusters with different charge states possess diverse properties including magnetism, ${ }^{10,11}$ optical absorption, ${ }^{8,9}$ catalytic activity ${ }^{12}$ and stability. ${ }^{13,14}$ Furthermore, Maran and co-workers studied the reduction or oxidation of $\left[\mathrm{Au}_{25} \mathrm{SR}_{18}\right]^{0}$ and evidenced a series of charge states, $-2,-1,+1,+2$, and +3 , under cyclic voltammetry. ${ }^{15}$

Whetten, Tsukuda and coworkers further investigated the effect of charge on the stability of $\mathrm{Au}_{25} \mathrm{SR}_{18} .{ }^{8}$ By judiciously chosing organic ligands lacking protonation or deprotonation sites, they managed to change the net charge on the cluster the core, while preserving the magic stability of the cluster. ${ }^{16-18}$ These results suggest that the magic stability of $\mathrm{Au}_{25}(\mathrm{SR})_{18}$, is attributable to geometric rather than electronic factors. Clearly, electrospray ionization mass spectrometry (ESI-MS) offered the opportunity to accurately map the charge state of $\operatorname{Au}_{25}(\mathrm{SR})_{18}$ nanoclusters. ${ }^{16-20}$ Mass spectrometry analysis of gold clusters after electrospray ionization usually yields ionic species with different charge states, depending on the propensity of the ligand to bear charges, most of the time through protonation or deprotonation. In this case, the observed species are of the form $\left[\mathrm{Au}_{\mathrm{n}} \mathrm{SR}_{\mathrm{n}} \pm \mathrm{mH}\right]^{ \pm z}$. In such species, part of the charge is borne by ligand moieties, through protonation or deprotonation, but the gold cluster itself can also be charged. Deconvolution of the isotope-resolved mass spectrum can then yield information on the charge state of the cluster, which can be further interpreted in terms of the oxidation state. ${ }^{19}$

Recently, using first principles calculations, the effects of charge state and type of ligands on the structural, electronic, and optical properties of the $\operatorname{Au}_{25}(\mathrm{SR})_{18}$ 
nanocluster were investigated. ${ }^{21}$ It was shown that distortions of the metal core become more pronounced when the charge state of the nanocluster increases (i.e. going from the anionic, to neutral, to cationic cluster) affecting optical spectra.

In addition to mass spectrometry, novel and complementary information on the intrinsic stability and structures of nanoclusters can be obtained by applying gasphase methods, ${ }^{22}$ including ion mobility mass spectrometry. Ion mobility-massspectrometry (IMS-MS) has also been used to gain insight in the structural diversity of nanoclusters, ${ }^{23-28}$ and on the influence of the charge state of cluster ions on their structure. ${ }^{20}$ An advantage of IMS and MS in the present context is their ability to isolate and study separately the different species that may co-exist in the sample.

In the present study, we used high resolution IMS and MS measurements to investigate the charging patterns of gold clusters ligated by mercaptobenzoic acid (MBA). Protons can be removed from MBA carboxyl groups. In the negative mode of ionization, the charging patterns can thus be a combination of the net charge of the core and the charge of the deprotonated ligands. In a previous study, we reported the influence of the ligand structure on the stability of the clusters by comparing $\mathrm{Au}_{25} \mathrm{MBA}_{18}$ clusters composed with different substitution isomers of MBA (ortho, meta, and para). ${ }^{20}$ In the following, we show that deconvolution of the mass spectra obtained for those clusters reveals that different charging patterns co-exist. Based on IMS separation of these populations and IMS-IMS-MS measurements, we give evidence that spontaneous electron loss occurring in the gas phase accounts for a large part of the observed diversity.

\section{Experimental Section}

\section{Materials}

Tetrachloroauric(III) acid trihydrate $\left(\mathrm{HAuCl}_{4} \cdot 3 \mathrm{H}_{2} \mathrm{O}\right)$, 4-mercaptobenzoic acid( $p$ MBA), and 3-mercaptobenzoic acid ( $m$-MBA) were obtained from Acros Organics; thiosalicylic acid (2-mercaptobenzoic acid, o-MBA), tributylamine, and ammonium acetate were purshased from Sigma-Aldrich; borane-trimethylamine complex was obtained from Alfa Aesar; and methanol and diethyl ether were obtained from VWR. Milli-Q water with a resistivity of $18.2 \mathrm{M} \Omega \mathrm{cm}$ was used for all experiments. 


\section{Synthesis of $\mathrm{Au}_{25} \mathrm{MBA}_{18}$ Clusters}

Similar synthetic conditions were used for the three (para, meta, or ortho) clusters. These conditions are thoroughly described in ref. 20. Briefly, mercaptobenzoic acid is dissolved in methanol and tributylamine, and tetrachloroauric acid trihydrate is added to form a gold thiolate/tributylamine complex. A slow reduction of gold is then obtained by adding trimethylamine borane. The mixture is finally left under stirring for $24 \mathrm{~h}$.

\section{IMS-MS and IMS-IMS-MS measurements}

High resolution IMS-MS measurements were done using a home-built IMS-IMSMS setup (see scheme S1 in supporting information) described in details in ref. 29 Solutions of $\mathrm{Au}_{25} \mathrm{MBA}_{18}$ were prepared at a concentration of $50 \mu \mathrm{mol} . \mathrm{L}^{-1}$ in ultrapure water and electrosprayed directly in the negative ion mode. We used the electrospray ion source of a Maxis Impact quadrupole-time-of-flight instrument (Bruker, Bremen, Germany), and the source conditions were adapted to minimize cluster fragmentation (see Supplementary Information). In our instrument, ions cross two identical drift tubes before being detected as a function of their massto-charge $(\mathrm{m} / \mathrm{z})$ ratio by a time-of-flight mass spectrometer (Maxis Impact Bruker, Bremen, Germany). This configuration allows to record mass spectra as a function of the drift time of the ions across the drift tubes. Arrival time distributions (ATDs) can then be extracted for species in $\mathrm{m} / \mathrm{z}$ ranges of interest. The arrival time of the ions depends on the ratio of their orientationally-averaged collision integral with the drift gas (or CCS), $\Omega$, and their charge $q::^{30}$

$$
t_{d}=\frac{16}{3} L \sqrt{\mu \frac{k_{B} T}{2 \pi}} \frac{N}{E} \frac{\Omega}{q}
$$

where $t_{d}$ is the time through a drift region of length $L, k_{B}$ is the Boltzmann constant, $T$ is the drift temperature, $\mathrm{E}$ is the drift field, $N$ is the number density of the buffer gas, and $\mu$ is the reduced mass for ion-neutral collisions.

The two drift tubes, DT1 and DT2, are $79 \mathrm{~cm}$ long. For the present experiments, the drift region was filled with 4 Torr helium maintained at room temperature (298 K). A constant DC electric field of $6.3 \mathrm{~V} \cdot \mathrm{cm}^{-1}$ was applied across each tube. As depicted in Scheme S1, three dual ion funnel assemblies, FA, FB, and FC separate the different regions of the instrument. They are used to ensure for good 
transmission of the ions. FA and FB also serve as ion traps and can be used to inject short (300 $\mu \mathrm{s}$ ) ion bunches in DT1 or DT2, respectively, thus defining a reference for $\mathrm{t}=0$ in IMS arrival time measurements. This dual tube configuration allows different operation modes.

\section{IMS measurements}

For high-resolution IMS measurements, ion bunches are injected from FA and a DC gradient (typically 6-8 V.cm-1) is applied across FB to prevent ions from being trapped in this region. Arrival times are then measured using DT1 and DT2 as a single drift tube. This mode allows to achieve high IMS resolution by taking advantage of a longer drift path, and a higher drift voltage. The resolving power for IMS separation is limited by diffusion and scales with the square root of the drift voltage as: ${ }^{30}$

$$
R_{\text {diff }}=\frac{t_{d}}{\Delta t_{d}}=\frac{1}{4 \sqrt{\ln (2)}} \sqrt{\frac{q V}{k_{B} T}}
$$

where $\Delta t_{d}$ is the FWHM of a diffusion-limited IMS peak, $k_{B}$ is the Boltzmann constant, $T$ is the drift temperature, $q$ is the charge of the ions, and $V$ is the voltage across the drift cell. In the present experimental conditions, using drift voltage of $1000 \mathrm{~V}$ across DT1+DT2, the theoretical resolving power for ions with 5 charges is then $R_{\text {diff }}=132$. In practice, the finite duration of the injected ion bunch limits the effective experimental resolving power to 80-90 for the ions of interest. In the experimental ATDs recorded in this operation mode, the arrival time refers to the injection in DT1.

\section{IMS-IMS measurements}

In the IMS-IMS operation mode, the ion gate at the end of DT1 is used to cut a "slice" in the ATD, thus letting through only the ions in a narrow arrival time range. This allows isomer selection. ${ }^{31-33}$ The ATDs recorded in this mode gives information about potential modifications of the ions after selection. Namely, an ATD not consisting in a single peak centered at the selected drift time provides a signature of a change in the mobility of the ions, i.e. a change in their "surface-to-charge" ratio. This procedure was previously used to study isomerization, corresponding to a change of CCS, 34,35 but it is also clear from equation (1), that a change in the charge $q$ also affects the arrival time. 


\section{MS-MS measurements}

Classical collision induced dissociation experiments were performed using the quadrupole of the Maxis Impact to select precursor ions, and the time-of-flight to detect fragments. Selected ions were subjected to collisional activation by varying the relative bias voltage of the quadrupole and the collision cell. We used dry $\mathrm{N}_{2}$ as a collision gas. The following instrument settings were used (as defined in Bruker o-tofControl software): collision gas flow: $22 \%$, quadrupole isolation width: $\pm 5 \mathrm{~m} / \mathrm{z}$.

\section{Deconvolution of the mass spectra}

The isotope distributions of the ions of interest were fitted using homemade python software based on the SciPy library (version 1.2.1). ${ }^{36}$ Our goal was to determine the relative populations of species with close chemical compositions (typically differing by one or two protons), thus displaying overlapping isotope distributions. The molmass package ${ }^{37}$ was used to compute theoretical isotope distributions for the different considered compositions. Theoretical isotopic distributions for the mixture were generated from a linear combination of the isotope distributions of the different considered compositions. An isotope pattern with Gaussian peaks was then computed from those distributions. This simulated pattern was finally adjusted to the experimental data, through minimization of the RMSD, leaving the weights of the different populations as only free fitting parameters. These weights were then interpreted as the relative populations of the different species detected for each charge state.

It important to note that the masses of the different candidate compositions are shifted by only one mass unit. The intensity of the peak at the lowest $\mathrm{m} / \mathrm{z}$ value then only corresponds to the species with the lowest mass (this is the monoisotopic peak for this species). We took advantage of this particularity by using a stepwise fitting procedure. The weight for the lowest mass species was first adjusted to fit the first mass peak. Then the weight for the second lowest mass species was adjusted to fit the second mass peak, keeping the first weight constant, etc.

\section{Results and Discussion}

Different populations with distinct charging patterns co-exist. Mass spectra were recorded in the negative electrospray mode for $\mathrm{Au}_{25} \mathrm{MBA}_{18}$ samples prepared 
with MBA ligands substituded either in ortho, meta, or para position. As previously reported, ${ }^{20}$ these spectra are dominated by peaks corresponding to the different charge states of $\mathrm{Au}_{25} \mathrm{MBA}_{18}$. For the three isomers of mercaptobenzoic acids (para/meta/ortho-MBA), the observed charge states range from $z=-3$ to $z=-5$ (data not shown). Higher charge states are nevertheless observed for para-MBA and, to a lesser extent, for meta-MBA containing clusters. ${ }^{20}$

We focused on the isotope distribution on the mass peaks of the different charge states of $\mathrm{Au}_{25} \mathrm{MBA}_{18}$ clusters. Figure 1 displays the isotope pattern for the 5- charge state of $\mathrm{Au}_{25}$ ortho- $\mathrm{MBA}_{18}$ clusters. The observed proton distribution could not be reproduced by considering a unique number $\mathrm{p}$ of deprotonation, using a raw formula of the form $\left[\mathrm{Au}_{25} \text { ortho- } \mathrm{MBA}_{18}-\mathrm{nH}\right]^{-5}$. Namely, the peak at the lowest $\mathrm{m} / \mathrm{z}$ in the pattern corresponds to the monoisotopic mass expected for $\left[\mathrm{Au}_{25}\right.$ ortho$\left.\mathrm{MBA}_{18}-6 \mathrm{H}\right]^{-5}$ ions. However, the intensity ratio of the different peaks of the isotope pattern are not well reproduced based on this composition. Using the abovedescribed fitting procedure, we determined that the observed isotope pattern was resulting from the coexistence of species with different raw formulas, including $\left[\mathrm{Au}_{25} \text { ortho- } \mathrm{MBA}_{18}-6 \mathrm{H}\right]^{-5}$, but also $\left[\mathrm{Au}_{25} \text { ortho- } \mathrm{MBA}_{18}-5 \mathrm{H}\right]^{-5}$ and $\left[\mathrm{Au}_{25}\right.$ ortho$\left.\mathrm{MBA}_{18}-4 \mathrm{H}\right]^{-5}$ in respective proportions of $51.0 \%, 30.9 \%$, and $18.1 \%$. The same deconvolution procedure was applied to all detected charge states for all investigated species, always yielding combinations of populations with different protonation states (see Figure S1).

In negative electrospray mode, deprotonation is a standard way of formation of charged species. In the present clusters, the carboxyl groups of the 18 MBA ligands are the most obvious deprotonation sites. It then seems reasonable to assume that a species with $n$ missing protons can accommodate $n$ charges on deprotonated carboxylate groups. For a given charge state $z$, the present deconvolution yields species of general formula $\left[\mathrm{Au}_{25} \text { ortho- } \mathrm{MBA}_{18}-\mathrm{nH}\right]^{-z}$. If $\mathrm{n} \neq \mathrm{z}$, only part of the total charge can be attributed to the deprotonated carboxylate groups. In the following, we characterize the difference between the number of deprotonations and the total net charge by defining the charge shift $x=n-z$. This charge shift can be positive, negative or 0 . When it is not 0 , it corresponds to the amount of positive or negative charge which is not a priori borne by a deprotonated carboxylate group. This charge has either to be located in another region of the ligand, at the interface between the gold core and the ligands, at the cluster core, or delocalized. As 
depicted in the inset of Figure 2, for species whose composition follows the raw formula $\left[\mathrm{Au}_{25} \mathrm{MBA}_{18}-(\mathrm{z}+\mathrm{x}) \mathrm{H}\right]^{-z}$ the value of $\mathrm{x}$ completely defines the corresponding charge repartition scheme: $z+x$ negative charges borne by ligand carboxyl groups, and $x$ charges located elsewhere inside the cluster.

As summarized in the pie charts of Figure 2, populations displaying different charge shift, and then different charge repartition schemes, co-exist for all investigated clusters. Their relative proportion varies depending on the substitution of the ligands, and on the net charge state $z$ of the species. For the lowest detected charge states, the observed species mostly correspond to $x=-1$, as already reported. ${ }^{20}$ As charge state increases however, contributions from other populations become more significant. Interestingly, the evolution of the populations in not smooth but stepwise. In a first step, the population for $x=-1$ decreases to about $50 \%$ of the total ions. Then, for the highest observed charge state, $x=0$, or $x=+1$ dominate, and the proportion of $x=-1$ is dramatically reduced. This two-step evolution is observed for the three isomers of mercaptobenzoic acids, but the charge state at which the steps occur are different. Namely, for ortho-MBA only three charge states are observed showing significantly different populations. In contrast, the populations for para-MBA remain similar up to charge state $z=-5$, and up to $z=-4$ for meta-MBA. To summarize, significant changes in the charge repartition scheme are observed only for the highest two charge states detected for each isomeric species.

The stability of the clusters is affected by their charging pattern. Previously reported $\mathrm{CID}$ experiments on the 4- charge states of $\mathrm{Au}_{25} \mathrm{MBA}_{18}$ clusters emphasized the influence of the substitution of the MBA ligand on the stability of the clusters. Namely, the relative stabilities of the three considered species was found to be para>meta>ortho. ${ }^{20}$ In order to investigate the influence of the charging pattern on the stability of the clusters, we performed CID experiments. We focused on the 5- charge state of the investigated clusters, because it corresponds to a broad diversity of populations and it is detected for all ligand substitutions. The evolution of the intensity of the detected fragments are plotted in Figure S2. Common fragmentation channels shared by the three considered ligand geometries include the apparition of $\left[\mathrm{Au}_{2} \mathrm{MBA}_{2}\right]^{-1}$, and the loss of $[\mathrm{AuMBA}]^{-}$ ${ }^{1}$ for the parent precursors. As already reported for the triply charged ions, clusters with ortho-MBA appear to fragment at significantly lower voltages that the other 
two. As a consequence, more species can be identified in the fragment mass spectra for these clusters, which can be attributed to the loss of up to two gold atoms, and up to five ligands. ${ }^{20}$

Based on the above-described deconvolution method, we extracted the evolution of the different populations identified within the precursor ion peak (e.g. $\left[\mathrm{Au}_{25}\right.$ $\left.\mathrm{MBA}_{18}-\mathrm{nH}\right]^{-z}$ with $\mathrm{x}=-1,0$ and +1 ) as a function of the collision voltage. The results are displayed on Figure 3. For clusters with para- and meta-MBA, no evolution is observed below a threshold voltage of $5 \mathrm{~V}$ and $9 \mathrm{~V}$ for meta- and para-, respectively. In contrast, the populations of clusters with ortho-MBA are modified yet at the lowest collision voltages, in relation with a decreased stability (see above). Once fragmentation is observed, the relative populations of species with different charge shifts are modified in each case. For meta- and para-substituted species, the populations corresponding to $x=-1$ are decreased relative to the others. This is also true for clusters with ortho-MBA, but a less pronounced decrease is also observed for the $x=0$ species. Interpreting those evolutions in terms of relative stability yields the conclusion that a more negative charge shift decreases the overall stability of the clusters.

\section{Evidence for heterogeneity within clusters with similar charging patterns.}

High-resolution arrival time distributions (ATDs) extracted for all detected species are represented in Figure 4. Most ATDs are dominated by a single spectral feature. However, the distributions for $\left[\mathrm{Au}_{25} \text { ortho-MBA}{ }_{18}\right]^{-5},\left[\mathrm{Au}_{25} \mathrm{meta}-\mathrm{MBA}_{18}\right]^{-5}$, and $\left[\mathrm{Au}_{25} \text { para-MBA} \mathrm{A}^{-6}\right]^{-6}$ clearly display two resolved peaks with comparable intensity. The width of these peaks correspond to the resolving power of the instrument (see Figure S3). CCS for charge states 3-, 4- and 5- were reported in ref. 20. In this work, we improved the resolution for CCS measurements, allowing for 2 different CCS values for $\left[\mathrm{Au}_{25} \text { ortho-MBA }{ }_{18}\right]^{-5}$, $\left[\mathrm{Au}_{25} \text { meta-MBA } \mathrm{MB}_{18}\right]^{-5}$, and $\left[\mathrm{Au}_{25} \text { para-MBA }{ }_{18}\right]^{-6}$ and we extended CCS measurements to charge states 6- and 7- (see Table S1 and Figure S4). We observe an increase of the CCS with charge state for clusters with para- and meta-MBA, which can be interpreted as a change in the ligand packing to accommodate Coulomb repulsion. No such change is observed for ortho-MBA, which reveals lower flexibility.

Beyond the most intense peaks, additional spectral features are also visible in most ATDs (see Figure 4). Firstly, the main peaks display a marked shoulder on the high drift time side in ATDs recorded for $\left[\mathrm{Au}_{25} \text { ortho-MBA }{ }_{18}\right]^{-5},\left[\mathrm{Au}_{25} \text { meta-MBA} A_{18}\right]^{-5}$, 
$\left[\mathrm{Au}_{25} \text { meta-MBA }{ }_{18}\right]^{-6}$ and $\left[\mathrm{Au}_{25} \text { para-MBA }{ }_{18}\right]^{-6}$. Secondly, a long tail to the short drift time side is also observed for $\left[\mathrm{Au}_{25} \text { ortho-MBA } 18\right]^{-4},\left[\mathrm{Au}_{25} \text { meta-MBA }{ }_{18}\right]^{-4},\left[\mathrm{Au}_{25}\right.$ para$\left.M B A_{18}\right]^{-5}$. In order to interpret these different spectral features, we took advantage of the possibility to extract from our raw data either the ATD for a single isotopic species, or conversely the mass spectrum corresponding to a selected arrival time range. On the left panel of Figure 5, we plotted the ATD for the peak at $\mathrm{m} / \mathrm{z} 1534.6$, i.e. the monoisotopic peak of $\left[\mathrm{Au}_{25} \text { meta-MBA } \mathrm{Al}_{18}-5 \mathrm{H}\right]^{-5}(\mathrm{x}=0)$. This monoisotopic ATD significantly differs from the full ATD obtained by integrating the whole isotopic pattern around $\mathrm{m} / \mathrm{z} 1535$, since the peak at $26.6 \mathrm{~ms}$ is not present. This observation suggests that the different features in the ATD may correspond to populations with different charging patterns. This is supported by the mass spectra on the right panel of Figure 5, which were obtained by integrating over different arrival time windows corresponding to the main spectral features observed in the full ATD, and denoted A, B, and C, in order of their respective arrival time. As expected from above, the extracted mass spectrum for peak $B$ reasonably matches with the expected isotope distribution for $\left[\mathrm{Au}_{25} \mathrm{meta}-\mathrm{MBA}_{18}\right.$ $5 \mathrm{H}]^{-5}(\mathrm{x}=0)$. Integrating across peak $A$ yields a mass spectrum shifted to higher $\mathrm{m} / \mathrm{z}$, which corresponds to a majority of $\left[\mathrm{Au}_{25} \text { meta-MBA} \mathrm{A}_{18}-4 \mathrm{H}\right]^{-5}(\mathrm{x}=-1)$. We interpret the minor peak at $\mathrm{m} / \mathrm{z} 1534.6$ in the projected mass spectrum for $A$ to partial overlap with peak $B$. The isotope pattern obtained for peak $C$ is more complex and has to be considered as resulting from the coexistence of different populations with different charge shifts ranging from -1 to +1 . Similar observations can be done from the deconvolution of the other multimodal ATDs (Figure S5). Interestingly, different populations with the same charge shift can be found at significantly different arrival times. This suggests that, beyond the heterogeneity of the clusters in terms of charging patterns, cluster with the same extra charge can also have different mobilities, and then possibly different structures. The existence of structural heterogeneity, in relation with different distributions of the net charge, was indeed recently predicted from first principles calculations. ${ }^{21,38,39}$

\section{Deconvolution of the ATDs reveals that clusters can lose electrons in the}

gas phase. Spectral features in an ATD, including tails, and even peaks, should not always be assigned to different structural families. It was shown that isomerization occurring on timescales smaller than or similar to that of the separation can yield characteristic signatures..$^{35,40,41}$ In this context, it is striking 
that the long tailing peaks to the short drift time side observed for $\left[\mathrm{Au}_{25}\right.$ ortho$\left.\mathrm{MBA}_{18}\right]^{-4}$, $\left[\mathrm{Au}_{25} \text { meta- } \mathrm{MBA}_{18}\right]^{-4}$, and $\left[\mathrm{Au}_{25} \text { para-MBA}{ }_{18}\right]^{-5}$ extend towards arrival times corresponding to that of $\left[\mathrm{Au}_{25} \mathrm{Ortho}-\mathrm{MBA}_{18}\right]^{-5},\left[\mathrm{Au}_{25} \text { meta-MBA}{ }_{18}\right]^{-5}$, and $\left[\mathrm{Au}_{25}\right.$ para$\left.\mathrm{MBA}_{18}\right]^{-6}$, respectively. Such coincidence would be consistent with a transformation occurring during the drift. Namely, ions starting their drift as $\left[\mathrm{Au}_{25} \text { meta-MBA } \mathrm{M}_{18}\right]^{-5}$ and losing an electron would be detected as $\left[\mathrm{Au}_{25} \text { meta-MBA} \mathrm{A}_{18}\right]^{-4}$. However, their

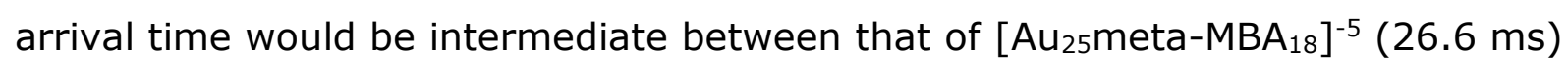
and $\left.\left[\mathrm{Au}_{25} \text { meta-MBA}\right]_{18}\right]^{-4}(32.6 \mathrm{~ms})$. This would result in the ATD of $\left[\mathrm{Au}_{25} \mathrm{meta}-\right.$ $\left.\mathrm{MBA}_{18}\right]^{-4}$ displaying a long tail, as that presently observed.

To test this hypothesis, we performed IMS-IMS-MS measurements by selecting $\left.\left[\mathrm{Au}_{25} \text { meta-MBA}\right]_{18}\right]^{-5}$ ions at the end of the first drift tube of our instrument. The ATDs recorded after selection for both $\left[\mathrm{Au}_{25} \text { meta- } M B A_{18}\right]^{-5}$ and $\left[\mathrm{Au}_{25} \text { meta-MBA}{ }_{18}\right]^{-}$ 4 , as shown in Figure 6 . The fact that significant signal is detected for [ $\mathrm{Au}_{25} \mathrm{meta}-$ $\left.\mathrm{MBA}_{18}\right]^{-4}$ when the selection window is centered on drift times characteristic of $\left.\left[\mathrm{Au}_{25} \text { meta-MBA}\right]_{18}\right]^{-5}$ clearly demonstrates that electron loss has occurred. The recorded ATD for $\left[\mathrm{Au}_{25} \text { meta-MBA }{ }_{18}\right]^{-4}$ are composed of a broad plateau. The plateau starts at the arrival time of the selected $\left[\mathrm{Au}_{25} \text { meta-MBA}{ }_{18}\right]^{-5}$ ions and extends to longer drift times. Ions that have lost an electron during their flight are responsible for this plateau. Those ions have been travelling part of their way through the tube as $\left[\mathrm{Au}_{25} \mathrm{meta}-\mathrm{MBA}_{18}\right]^{-5}$, and the other part as $\left[\mathrm{Au}_{25} \text { meta-MBA}{ }_{18}\right]^{-}$ 4 , after electron loss. As a consequence, their drift time is intermediate between

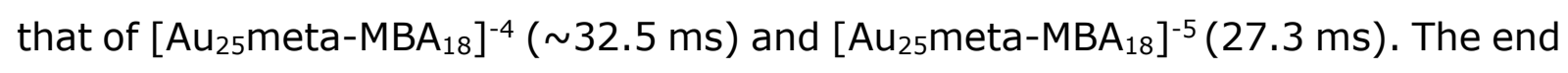
of the plateau corresponds to ions which have lost an electron immediately after selection and have then travelled all the way through DT2 as $\left[\mathrm{Au}_{25} \mathrm{meta}-\mathrm{MBA}_{18}\right]^{-4}$. Note that the apparent shift between the ends of the plateau in ATDs recorded with different selection windows is a simple consequence of the selection itself. As illustrated in Figure S6, the arrival times can be rescaled by subtracting the selection time in order to measure the drift time after selection. This way all plateaus end at the same time $(\sim 18.5 \mathrm{~ms})$, corresponding to the drift time of $\left[\mathrm{Au}_{25} \mathrm{meta}-\mathrm{MBA}_{18}\right]^{-4}$ through DT2 only.

Spontaneous electron loss in the gas phase was already observed for multiplycharged ions, ${ }^{42}$ and can be interpreted in terms of the Coulomb repulsion lowering the binding energy for an electron, potentially towards negative values. ${ }^{43}$ Tasaka et al. recently investigated electron photo-detachment in negatively charged $\mathrm{Ag}_{44}$ 
clusters with $\mathrm{SC}_{6} \mathrm{H}_{3} \mathrm{~F}_{2}$ ligands. ${ }^{44}$ They reported that such clusters were metastable yet at the 4- charge state, with a repulsive Coulomb barrier of $2.7 \mathrm{eV}$. We estimated the effect of Coulomb repulsion in our case, through a simple model for charges on a sphere. We considered $z$ charges distributed on a sphere of a radius compatible with that of the clusters (we estimated the radius $R$ from the average CCS of the clusters by $R=\sqrt{\frac{\langle C C S\rangle}{\pi}}$, yielding $12 \AA$ ). The optimal charge repartition was determined by numerically solving Thomson's problem for charges on a sphere, using the optimize.minimize routine from the Scipy library. ${ }^{36}$ Two charge distribution models were considered: all charges on the surface, or one charge at the center of the sphere. The Coulomb energy at each charged site was finally computed. We plotted the maximum value of these per-site energies as a function of $z$ in Figure 7. This simplistic model yields repulsive Coulomb energies comparable to the typical binding energy of the HOMO electron on a carboxylate $(\sim 3.5 \mathrm{eV})^{45}$, as soon as 5-6 charges are present. Although this model does not give any information on the energy barrier for electron detachment, it provides a rough estimate of the number of charges beyond which an electron may have a negative binding energy, thus being potentially able to spontaneously detach. Under this respect, the threshold around 5-6 charges is in good agreement with the interpretation of our experimental observations in terms of electron loss.

The ATDs in Figure 6.b have been normalized to the total ion signal (i.e. the sum of the intensities for $\left[\mathrm{Au}_{25} \text { meta- } M \mathrm{MBA}_{18}\right]^{-5}$ and $\left[\mathrm{Au}_{25} \text { meta-MBA}{ }_{18}\right]^{-4}$ ions). This allows the intensities recorded after selection of the two IMS peaks ( $A$ and $B$ in Figure 6 ) to be compared. It clearly appears that the signal for $\left[\mathrm{Au}_{25} \mathrm{meta}^{-} \mathrm{MBA}_{18}\right]^{-4}$ is significantly higher after selection of the region $\mathrm{B}$. More precisely, the ratio of the ion signal for $\left[\mathrm{Au}_{25} \mathrm{meta}^{-} \mathrm{MBA}_{18}\right]^{-4}$ to the total ion signal, which can be interpreted as a detachment yield is $13 \%$ and $27 \%$ after selection of $A$ and $B$ regions, respectively. If we consider the average drift time after selection, the corresponding lifetimes can be estimated to be $\tau_{A}=118 \mathrm{~ms}$ and $\tau_{B}=54 \mathrm{~ms}$. This difference further supports that the $\left[\mathrm{Au}_{25} \text { meta-MBA}{ }_{18}\right]^{-5}$ ion populations separated by IMS correspond to different structures, and possibly different charge repartition patterns. Based on the energetics of Coulomb repulsion included in the above model, the population in the A region, with a more compact structure, is also expected to display the lowest electron binding energy. We nevertheless observe slower electron loss from this population. This suggests that the detachment 
mechanisms for populations A and B may involve different electronic states, and possibly electrons localized in different regions of the cluster. Indeed, the extra charge for population $B$ is $x=0$, while population $A$ is dominated by $x=-1$. On the other hand, the dynamics for electron loss may also be limited by other factors, such as the existence of intermediate states involving a geometrical deformation of the cluster or the ligands before detachment. This would also be consistent with the timescales involved. Moreover, the tail on the long arrival times side on the ATDs for $\left[\mathrm{Au}_{25} \text { meta-MBA} 18\right]^{-5}$ ions (Figure 6.a) would be consistent with the latter hypothesis.

Consequences of electron loss on the observed charging patterns. The existence of electron detachment provides new insight in the above-described heterogeneities in the charging pattern of the clusters. After a cluster has lost an electron, its charge shift is increased by 1 . As illustrated in Scheme 1, this implies that part of the population with a charge shift $x=0$ at charge state $z$ may originate from clusters initially bearing $z+1$ negative charges (with a charge shift of -1 ), which have lost an electron before being detected. As a consequence, the population detected with an extra charge $x=0$ is a mixture of clusters natively displaying a 0 charge shift, and clusters natively displaying a -1 charge shift and having lost an electron.

Clusters with the same charge shift may then be detected at different arrival times (see Figure 5 and Figure S5) depending on whether they have lost an electron or not. Beyond the kinetic effects discussed in the above section, different arrival times generally correspond to different CCS. It is indeed likely that electron loss results in a structural change (then a change in CCS). A higher net charge generally results in a higher CCS (Figure S4). As already mentioned in the above section, electron loss may then be accompanied by partial structural re-organization of the cluster, especially of the ligands, due to the release of Coulombic repulsion. However, the timescale necessary for such structural relaxation is difficult to estimate, and long-lived metastable structural intermediates may be involved. On the other hand, even if complete structural relaxation occurs after electron loss, charges are most probably located on different sites for different populations of clusters. This also results in different CCSs.

A detailed interpretation of the complex processes that may be triggered by the loss of an electron nevertheless lies out of the scope of the present paper. Let us 
rather focus on the consequence of electron loss on the measured charge shift distributions summarized in Figure 2. In particular, the contribution of electron loss allows tentative interpretation of the observation that clusters with a -1 charge shift dominate for the lowest charge states, whereas clusters at higher charge states mainly display a 0 charge shift. The populations we observe are affected by the time-window of the detection. In other words, we see only species that survive long-enough to be detected. Our CID results (Figure 3 ) emphasized the decreased stability of clusters with -1 charge shift as compared to those that with $x=0$ or +1 . Moreover our toy model for Coulomb repulsion suggests that part of the charge located in the vicinity of the cluster core would result in an increase of the Coulomb repulsion at the peripheral charged sites (see Figure 7), and then favor electron detachment, even if other factors also influence electron loss kinetics. Clusters with a charge shift $x=-1$ may then not be stable enough to survive as highly charges species, even though such species are formed during the electrospray process.

Differences in lifetimes would then explain that species with $x=0$ (even $x=+1$, for ortho-MBA) dominate for the highest observed charge states. Under the present hypothesis, those clusters would mainly originate from metastable species with higher charge states, and a more negative charge shifts. Furthermore, the absence of higher charges states in the mass spectra (especially for clusters with orthoMBA) is consistent with this explanation, considering that those species are not stable during the timescale of the experiment. Importantly, as illustrated by our spherical model in Figure 7, the decrease of the Coulomb repulsion energy after electron loss (about $0.8 \mathrm{eV}$ per electron) is expected to be important enough to significantly increase the lifetime of the resulting species. Therefore, no more than two electron loss events are expected to occur within the timescale of the experiment. It is thus likely that part of the heterogeneity in the extra charge of the clusters reflects that different charging patterns pre-exist in solution. This is potentially the case for the observed proportion (about $10 \%$ ) of clusters with $x=0$ for the lowest charge states of meta- and para-MBA containing clusters. Moreover, the presence of clusters with $x=-2$, for clusters with ortho-MBA at charge state 3 cannot result from an artifact due to electron loss. 


\section{Conclusions}

Mass spectrometry and ion mobility spectrometry were used to investigate the heterogeneity in the charging patterns of $\mathrm{Au}_{25} \mathrm{MBA}_{18}$ clusters. We showed that for a given charge state of those clusters, several populations with different protonation patterns co-exist. Those populations display different stabilities with respect to collisional activation. Ion mobility experiments also demonstrate that they have different cross sections, and then display not only different charge locations, but also possibly different structures. However, no systematic correlation was found between the amount of charges not attributable to deprotonation and the CCS. In particular, the present results do not allow to emphasize potential charge-driven deformations of the cluster core. From the deconvolution of the ion mobility distributions, and based on tandem-ion mobility measurements, we gave evidence that part of the heterogeneity in the charging patterns of the clusters was attributable to the existence of spontaneous electron loss during the measurement. This effect has to be taken into account to avoid biases in data interpretation, especially when considering the highest charge states that are detected. Heterogeneities in structure and charging patterns are nevertheless present even for charge states that are not likely affected by the above-cited bias. This suggests that such heterogeneity pre-exists in solution, possibly in relation with different oxidation states of the cluster. Numerous wet chemistry strategies have been proposed to design atomically precise metal clusters, with remarkable monodispersity in terms of composition. The present work provides further evidence that other sources of dispersity have to be considered, which may significantly affect the physical and chemical properties of the clusters.

Supporting information: Scheme of the experimental setup, deconvoluted mass spectra for all investigated species, collision-induced fragmentation results, experimental CCS values, arrival-time-specific mass spectra for $\left[\mathrm{Au}_{25}\right.$ ortho$\left.\mathrm{MBA}_{18}\right]^{-5}$ and $\left[\mathrm{Au}_{25} \text { para-MBA}{ }_{18}\right]^{-6}$, rescaled IMS-IMS ATDs. 


\section{References}

(1) Jin, R. Quantum Sized, Thiolate-Protected Gold Nanoclusters. Nanoscale 2010, 2 (3), 343-362. https://doi.org/10.1039/B9NR00160C.

(2) Zeng, C.; Chen, Y.; Das, A.; Jin, R. Transformation Chemistry of Gold Nanoclusters: From One Stable Size to Another. J. Phys. Chem. Lett. 2015, 6 (15), 2976-2986. https://doi.org/10.1021/acs.jpclett.5b01150.

(3) Jin, R.; Zeng, C.; Zhou, M.; Chen, Y. Atomically Precise Colloidal Metal Nanoclusters and Nanoparticles: Fundamentals and Opportunities. Chem. Rev. 2016, 116 (18), 10346-10413. https://doi.org/10.1021/acs.chemrev.5b00703.

(4) Chakraborty, I.; Pradeep, T. Atomically Precise Clusters of Noble Metals: Emerging Link between Atoms and Nanoparticles. Chem. Rev. 2017, 117 (12), 8208-8271. https://doi.org/10.1021/acs.chemrev.6b00769.

(5) Kang, X.; Chong, H.; Zhu, M. $\operatorname{Au}_{25}(\mathrm{SR})_{18}$ : The Captain of the Great Nanocluster Ship. Nanoscale 2018, 10 (23), 10758-10834. https://doi.org/10.1039/C8NR02973C.

(6) Zhu, M.; Aikens, C. M.; Hollander, F. J.; Schatz, G. C.; Jin, R. Correlating the Crystal Structure of A Thiol-Protected $\mathrm{Au}_{25}$ Cluster and Optical Properties. J. Am. Chem. Soc. 2008, 130 (18), 5883-5885. https://doi.org/10.1021/ja801173r.

(7) Heaven, M. W.; Dass, A.; White, P. S.; Holt, K. M.; Murray, R. W. Crystal Structure of the Gold Nanoparticle $\left[\mathrm{N}\left(\mathrm{C}_{8} \mathrm{H}_{17}\right)_{4}\right]\left[\mathrm{Au}_{25}\left(\mathrm{SCH}_{2} \mathrm{CH}_{2} \mathrm{Ph}\right)_{18}\right]$. J. Am. Chem. Soc. 2008, 130 (12), 3754-3755. https://doi.org/10.1021/ja800561b.

(8) Negishi, Y.; Chaki, N. K.; Shichibu, Y.; Whetten, R. L.; Tsukuda, T. Origin of Magic Stability of Thiolated Gold Clusters: A Case Study on $\mathrm{Au}_{25}\left(\mathrm{SC}_{6} \mathrm{H}_{13}\right)_{18}$. J. Am. Chem. Soc. 2007, 129 (37), 11322-11323. https://doi.org/10.1021/ja073580+.

(9) Zhu, M.; Eckenhoff, W. T.; Pintauer, T.; Jin, R. Conversion of Anionic [Au25(SCH2CH2 Ph)18 ]- Cluster to Charge Neutral Cluster via Air Oxidation. J. Phys. Chem. C 2008, 112 (37), 14221-14224. 
https://doi.org/10.1021/jp805786p.

(10) Tofanelli, M. A.; Salorinne, K.; Ni, T. W.; Malola, S.; Newell, B.; Phillips, B.; Häkkinen, H.; Ackerson, C. J. Jahn-Teller Effects in $\mathrm{Au}_{25}(\mathrm{SR})_{18}$. Chem. Sci. 2016, 7 (3), 1882-1890. https://doi.org/10.1039/C5SC02134K.

(11) Zhu, M.; Aikens, C. M.; Hendrich, M. P.; Gupta, R.; Qian, H.; Schatz, G. C.; Jin, R. Reversible Switching of Magnetism in Thiolate-Protected Au 25 Superatoms. J. Am. Chem. Soc. 2009, 131 (7), 2490-2492. https://doi.org/10.1021/ja809157f.

(12) Chong, H.; Li, P.; Wang, S.; Fu, F.; Xiang, J.; Zhu, M.; Li, Y. Au 25 Clusters as Electron-Transfer Catalysts Induced the Intramolecular Cascade Reaction of 2-Nitrobenzonitrile. Sci. Rep. 2013, 3 (1), 3214. https://doi.org/10.1038/srep03214.

(13) Antonello, S.; Dainese, T.; Maran, F. Exploring Collective Substituent Effects: Dependence of the Lifetime of Charged States of $\mathrm{Au}_{25}\left(\mathrm{SC}_{n} \mathrm{H}_{2 n+1}\right)_{18}$ Nanoclusters on the Length of the Thiolate Ligands. Electroanalysis 2016, 28 (11), 2771-2776. https://doi.org/10.1002/elan.201600323.

(14) Collins, C. B.; Tofanelli, M. A.; Crook, M. F.; Phillips, B. D.; Ackerson, C. J. Practical Stability of $\mathrm{Au}_{25}(\mathrm{SR})_{18}-1 / 0 /+1 . R S C A d v .2017,7$ (71), 4506145065. https://doi.org/10.1039/C7RA07511A.

(15) Antonello, S.; Perera, N. V.; Ruzzi, M.; Gascón, J. A.; Maran, F. Interplay of Charge State, Lability, and Magnetism in the Molecule-like $\mathrm{Au}_{25}(\mathrm{SR})_{18}$ Cluster. J. Am. Chem. Soc. 2013, 135 (41), 15585-15594. https://doi.org/10.1021/ja407887d.

(16) Hamouda, R.; Bellina, B.; Bertorelle, F.; Compagnon, I.; Antoine, R.; Broyer, M.; Rayane, D.; Dugourd, P. Electron Emission of Gas-Phase $\left[\mathrm{Au}_{25}(\mathrm{SG})_{18^{-}}\right.$ $6 \mathrm{H}^{7-}$ Gold Cluster and Its Action Spectroscopy. J. Phys. Chem. Lett. 2010, 1 (21), 3189-3194. https://doi.org/doi: 10.1021/jz101287m.

(17) Negishi, Y.; Takasugi, Y.; Sato, S.; Yao, H.; Kimura, K.; Tsukuda, T. MagicNumbered $A u_{n}$ Clusters Protected by Glutathione Monolayers ( $\mathrm{n}=18,21$, 25, 28, 32, 39): Isolation and Spectroscopic Characterization. J. Am. Chem. Soc. 2004, 126 (21), 6518-6519. https://doi.org/10.1021/ja0483589. 
(18) Negishi, Y.; Nobusada, K.; Tsukuda, T. Glutathione-Protected Gold Clusters Revisited: Bridging the Gap between Gold(I)-Thiolate Complexes and Thiolate-Protected Gold Nanocrystals. J. Am. Chem. Soc. 2005, 127 (14), 5261-5270. https://doi.org/10.1021/ja042218h.

(19) Hamouda, R.; Bertorelle, F.; Rayane, D.; Antoine, R.; Broyer, M.; Dugourd, P. Glutathione Capped Gold $\mathrm{Au}_{\mathrm{N}}(\mathrm{SG})_{\mathrm{M}}$ Clusters Studied by Isotope-Resolved Mass Spectrometry. Int. J. Mass Spectrom. 2013, 335, 1-6. https://doi.org/10.1016/j.ijms.2012.10.008.

(20) Bertorelle, F.; Russier-Antoine, I.; Comby-Zerbino, C.; Chirot, F.; Dugourd, P.; Brevet, P.-F.; Antoine, R. Isomeric Effect of Mercaptobenzoic Acids on the Synthesis, Stability, and Optical Properties of $\mathrm{Au}_{25}(\mathrm{MBA})_{18}$ Nanoclusters. ACS Omega 2018, 3 (11), 15635-15642. https://doi.org/10.1021/acsomega.8b02615.

(21) Juarez-Mosqueda, R.; Mpourmpakis, G. Elucidating the Optical Spectra of $\left[\mathrm{Au}_{25}(\mathrm{SR})_{18}\right]^{\mathrm{q}}$ Nanoclusters. Phys. Chem. Chem. Phys. 2019, 21 (40), 22272-22282. https://doi.org/10.1039/C9CP03982A.

(22) Hirata, K.; Tomihara, R.; Kim, K.; Koyasu, K.; Tsukuda, T. Characterization of Chemically Modified Gold and Silver Clusters in Gas Phase. Phys. Chem. Chem. 2019, 21 (32), 17463-17474. https://doi.org/10.1039/C9CP02622C.

(23) Baksi, A.; Ghosh, A.; Mudedla, S. K.; Chakraborty, P.; Bhat, S.; Mondal, B.; Krishnadas, K. R.; Subramanian, V.; Pradeep, T. Isomerism in Monolayer Protected Silver Cluster Ions: An Ion Mobility-Mass Spectrometry Approach. J. Phys. Chem. C 2017, 121 (24), 13421-13427. https://doi.org/10.1021/acs.jpcc.7b04559.

(24) Comby-Zerbino, C.; Bertorelle, F.; Chirot, F.; Dugourd, P.; Antoine, R. Structural Insights into Glutathione-Protected Gold $\operatorname{Au}_{10-12}(\mathrm{SG})_{10-12}$ Nanoclusters Revealed by Ion Mobility Mass Spectrometry. Eur. Phys. J. D 2018, 72 (8), 144. https://doi.org/10.1140/epjd/e2018-90133-8.

(25) Harkness, K. M.; Fenn, L. S.; Cliffel, D. E.; Mclean, J. A. Surface Fragmentation of Complexes from Thiolate Protected Gold Nanoparticles by Ion Mobility-Mass Spectrometry. Anal. Chem. 2010, 82 (7), 3061-3066. 
https://doi.org/10.1021/ac100251d.

(26) Soleilhac, A.; Bertorelle, F.; Comby-Zerbino, C.; Chirot, F.; Calin, N.; Dugourd, P.; Antoine, R. Size Characterization of Glutathione-Protected Gold Nanoclusters in the Solid, Liquid and Gas Phases. J. Phys. Chem. C 2017, 121 (49), 27733-27740. https://doi.org/10.1021/acs.jpcc.7b09500.

(27) Comby-Zerbino, C.; Perić, M.; Bertorelle, F.; Chirot, F.; Dugourd, P.; Bonačić-Koutecký, V.; Antoine, R. Catenane Structures of Homoleptic Thioglycolic Acid-Protected Gold Nanoclusters Evidenced by Ion MobilityMass Spectrometry and DFT Calculations. Nanomaterials 2019, 9 (3), 457. https://doi.org/10.3390/nano9030457.

(28) Ligare, M. R.; Baker, E. S.; Laskin, J.; Johnson, G. E. Ligand Induced Structural Isomerism in Phosphine Coordinated Gold Clusters Revealed by Ion Mobility Mass Spectrometry. Chem. Commun. 2017, 53 (53), 73897392. https://doi.org/10.1039/C7CC02251D.

(29) Simon, A.-L.; Chirot, F.; Choi, C. M.; Clavier, C.; Barbaire, M.; Maurelli, J.; Dagany, X.; MacAleese, L.; Dugourd, P. Tandem Ion Mobility Spectrometry Coupled to Laser Excitation. Rev. Sci. Instrum. 2015, 86 (9), 094101. https://doi.org/10.1063/1.4930604.

(30) Revercomb, H. E.; Mason, E. A. Theory of Plasma Chromatography, Gazeous Electrophoresis - Review. Anal. Chem. 1975, 47 (7), 970-983. https://doi.org/10.1021/ac60357a043.

(31) Choi, C. M.; MacAleese, L.; Dugourd, P.; Choi, M. C.; Chirot, F. PhotoInduced Linkage Isomerization in the Gas Phase Probed by Tandem Ion Mobility and Laser Spectroscopy. Phys. Chem. Chem. Phys. 2018, 20 (17), 12223-12228. https://doi.org/10.1039/C8CP01833B.

(32) Choi, C. M.; Simon, A.-L.; Chirot, F.; Kulesza, A.; Knight, G.; Daly, S.; MacAleese, L.; Antoine, R.; Dugourd, P. Charge, Color, and Conformation: Spectroscopy on Isomer-Selected Peptide Ions. J. Phys. Chem. B 2016, 120 (4), 709-714. https://doi.org/10.1021/acs.jpcb.5b11919.

(33) Choi, C. M.; Kulesza, A.; Daly, S.; MacAleese, L.; Antoine, R.; Dugourd, P.; Chirot, F. Ion Mobility Resolved Photo- fragmentation to Discriminate Protomers. Rapid Commun. Mass Spectrom. 2019, 33 (S1), 28-34. 
https://doi.org/10.1002/rcm.8202.

(34) Koeniger, S. L.; Clemmer, D. E. Resolution and Structural Transitions of Elongated States of Ubiquitin. J. Am. Soc. Mass Spectrom. 2007, 18 (2), 322-331. https://doi.org/10.1016/j.jasms.2006.09.025.

(35) Poyer, S.; Comby-Zerbino, C.; Choi, C. M.; MacAleese, L.; Deo, C.; Bogliotti, N.; Xie, J.; Salpin, J.-Y.; Dugourd, P.; Chirot, F. Conformational Dynamics In Ion Mobility Data. Anal. Chem. 2017, 89 (7), 4230-4237. https://doi.org/10.1021/acs.analchem.7b00281.

(36) Virtanen, P.; Gommers, R.; Oliphant, T. E.; Haberland, M.; Reddy, T.; Cournapeau, D.; Burovski, E.; Peterson, P.; Weckesser, W.; Bright, J.; et al. SciPy 1.0: Fundamental Algorithms for Scientific Computing in Python. Nat. Methods 2020, 17 (3), 261-272. https://doi.org/10.1038/s41592-0190686-2.

(37) Gohlke, C. molmass 2020.1.1 https://pypi.org/project/molmass/ (accessed May 31, 2020).

(38) Lugo, G.; Schwanen, V.; Fresch, B.; Remacle, F. Charge Redistribution Effects on the UV-Vis Spectra of Small Ligated Gold Clusters: A Computational Study. J. Phys. Chem. C 2015, 119 (20), 10969-10980. https://doi.org/10.1021/jp511120j.

(39) Wing-Bocanegra, A.; Tlahuice-Flores, A. Effect of the Charge State on Bare Monoicosahedral $\left[\mathrm{Au}_{13}\right]^{\mathrm{Z}+}$ and Diphosphine-Protected $\mathrm{Au}_{13}$ Clusters $\left[\mathrm{Au}_{13}(\mathrm{Dmpe})_{5} \mathrm{Cl}_{2}\right]^{\mathrm{Z}+}$ : Structural, Electronic and Vibrational DFT Studies. Phys. Chem. Chem. Phys. 2019, 21 (43), 23855-23864. https://doi.org/10.1039/C9CP04827H.

(40) Wyttenbach, T.; Pierson, N. A.; Clemmer, D. E.; Bowers, M. T. Ion Mobility Analysis of Molecular Dynamics. Annu. Rev. Phys. Chem. 2014, 65 (1), 175196. https://doi.org/10.1146/annurev-physchem-040513-103644.

(41) Kinnear, B. S.; Hartings, M. R.; Jarrold, M. F. Helix Unfolding in Unsolvated Peptides. J. Am. Chem. Soc. 2001, 123 (24), 5660-5667. https://doi.org/10.1021/ja004196e.

(42) Wang, X.-B.; Wang, L.-S. Observation of Negative Electron-Binding Energy 
in a Molecule. Nature 1999, 400 (6741), 245-248. https://doi.org/10.1038/22286.

(43) Wang, L.-S.; Wang, X.-B. Probing Free Multiply Charged Anions Using Photodetachment Photoelectron Spectroscopy. J. Phys. Chem. A 2000, 104 (10), 1978-1990. https://doi.org/10.1021/jp9940093.

(44) Tasaka, Y.; Nakamura, K.; Malola, S.; Hirata, K.; Kim, K.; Koyasu, K.; Häkkinen, H.; Tsukuda, T. Electron Binding in a Superatom with a Repulsive Coulomb Barrier: The Case of $\left[\mathrm{Ag}_{44}\left(\mathrm{SC}_{6} \mathrm{H}_{3} \mathrm{~F}_{2}\right)_{30}\right]^{4-}$ in the Gas Phase. J. Phys. Chem. Lett. 2020, 11 (8), 3069-3074. https://doi.org/10.1021/acs.jpclett.0c00786.

(45) Antoine, R.; Joly, L.; Allouche, A. R.; Broyer, M.; Lemoine, J.; Dugourd, P. Electron Photodetachment of Trapped Doubly Deprotonated Angiotensin Peptides. UV Spectroscopy and Radical Recombination. Eur. Phys. J. D 2009, 51 (1), 117-124. https://doi.org/10.1140/epjd/e2008-00076-4. 


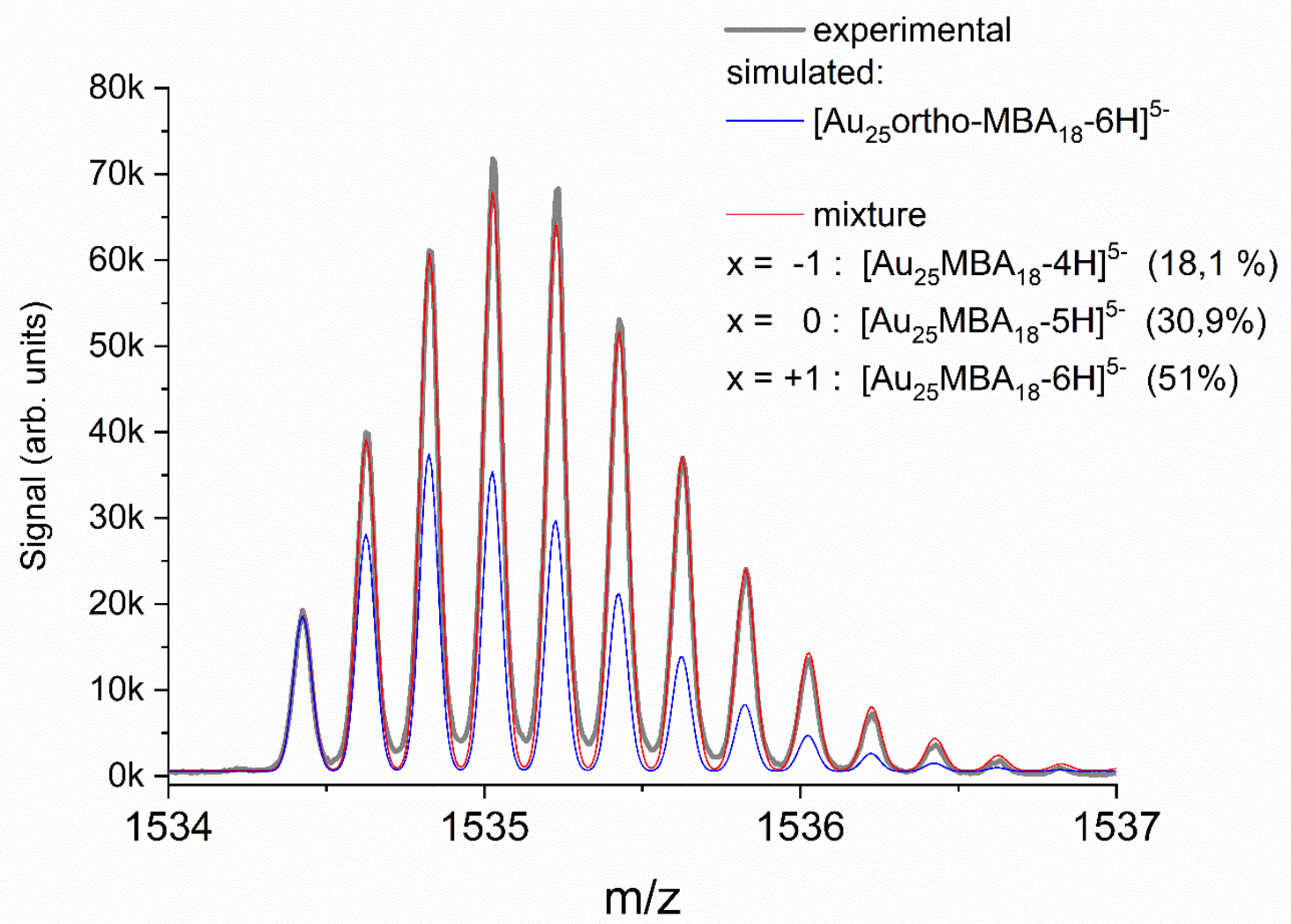

Figure 8: (black line) experimental isotope pattern for $\left[\mathrm{Au}_{25} \mathrm{Or} \text { tho- } \mathrm{MBA}_{18}\right]^{-5}$. The blue line corresponds to the simulated isotope patters for $\left[\mathrm{Au}_{25} \mathrm{Or} \text { tho-MBA } \mathrm{MB}_{18}-6 \mathrm{H}\right]^{-5}$ adjusted to fit the intensity of the experimental monoisotopic peak. The red line is a fit obtained by considering a mixture of species with different de-protonation patterns, corresponding to different charge shifts $x$. 

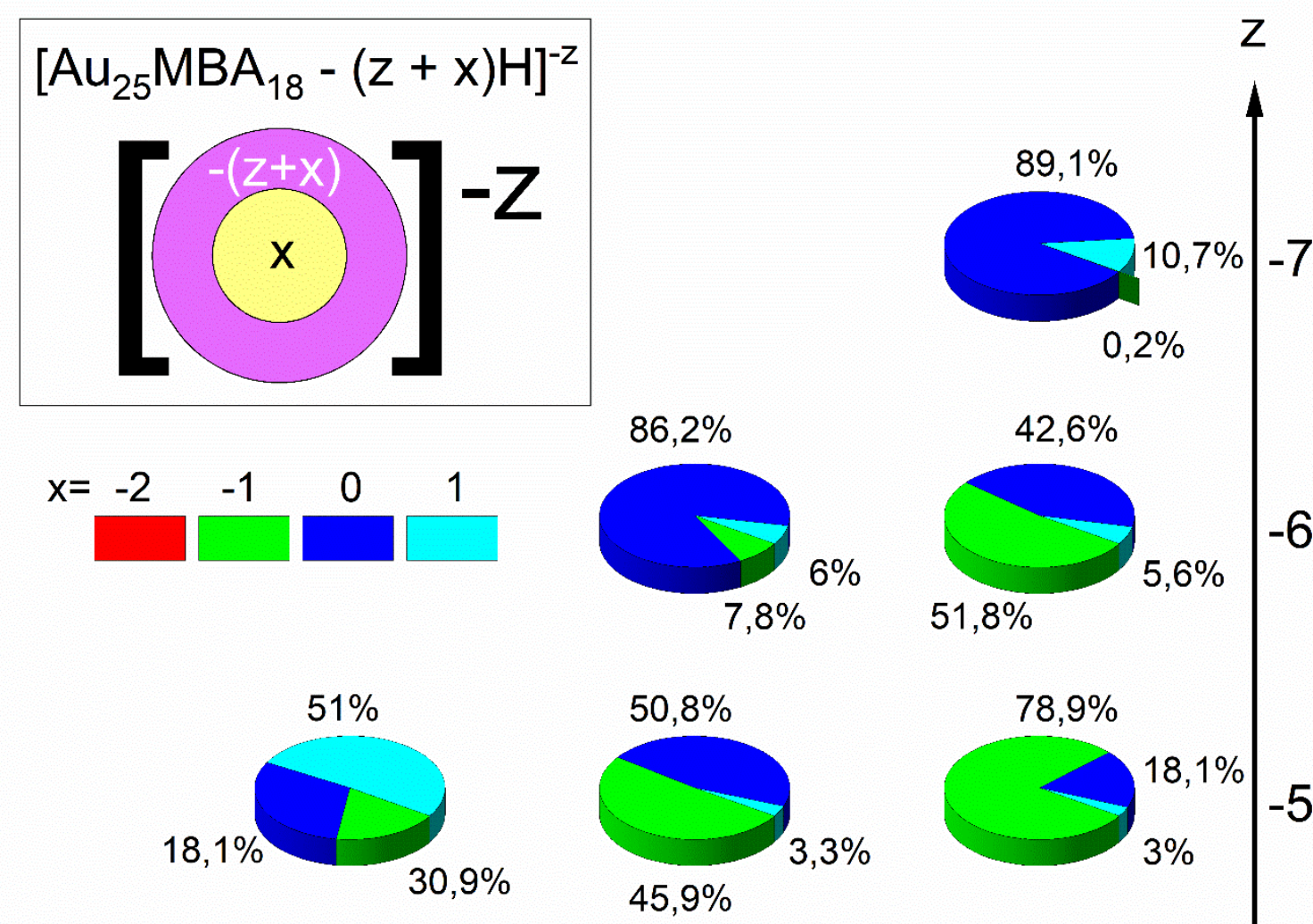

$18,1 \%$ $3 \%$ $-5$

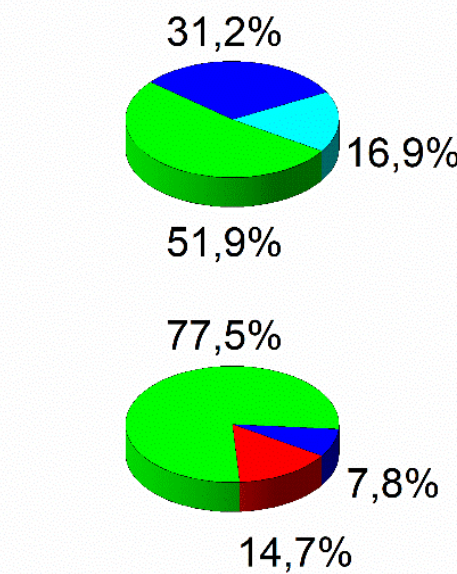

ortho

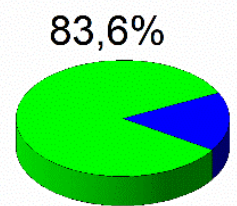

$16,4 \%$

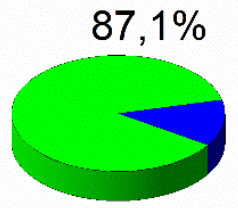

$12,9 \%$

$-4$

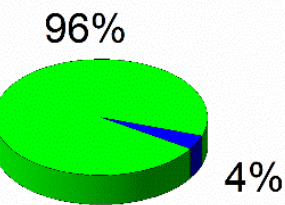

$4 \%$

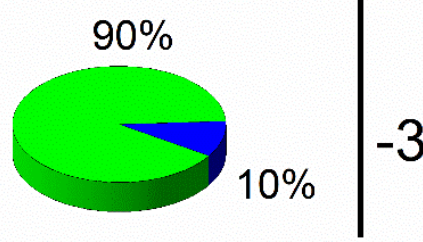

para

ligand

Figure 9: The inset illustrates the charge repartition corresponding to a charge shift $x$ for a cluster with total charge $-z$ : $z+x$ negative charges are borne by deprotonated ligand carboxyl groups, and the remaining $x$ charges are located elsewhere inside the cluster. $x$ can be positive or negative. The pie charts represent the relative proportions of populations exhibiting different charge 
repartitions, characterized by different values of $x$, as deduced from deconvolution of the experimental mass spectra.

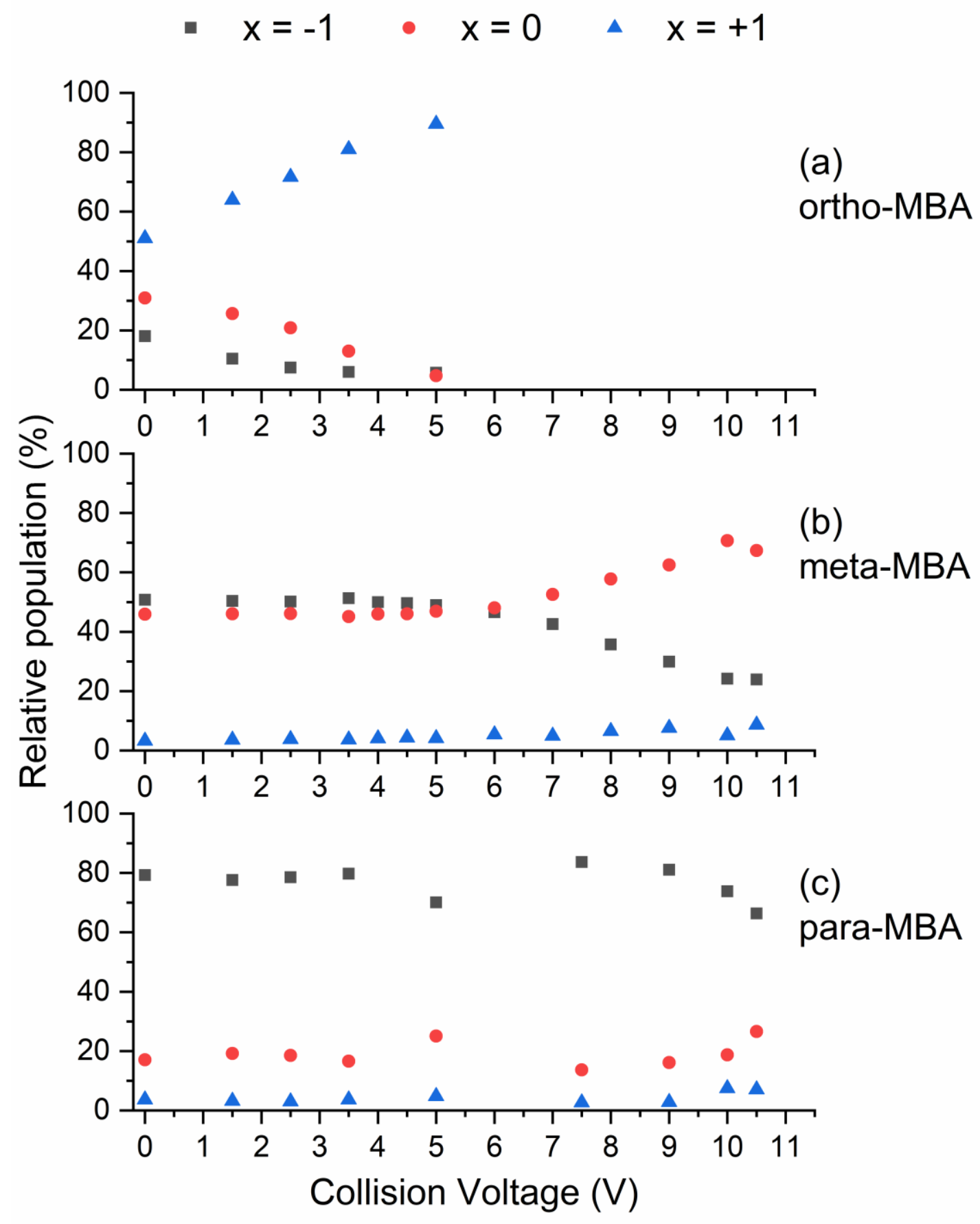

Figure 10: Evolution of the relative population of clusters with different charge shifts $\mathrm{x}$ in the precursor ion as a function of the collision voltage. Populations corresponding to $\mathrm{x}=0,-1$ and +1 are represented by red circles, black squares, and blue triangles, respectively. The selected precursor ion was the peak at 
$\mathrm{m} / \mathrm{z}$ 1536, corresponding to the 5- charge state of the clusters. Panels (a), (b), and (c) display the results obtained with ortho-, meta-, and para-MBA ligands, respectively.
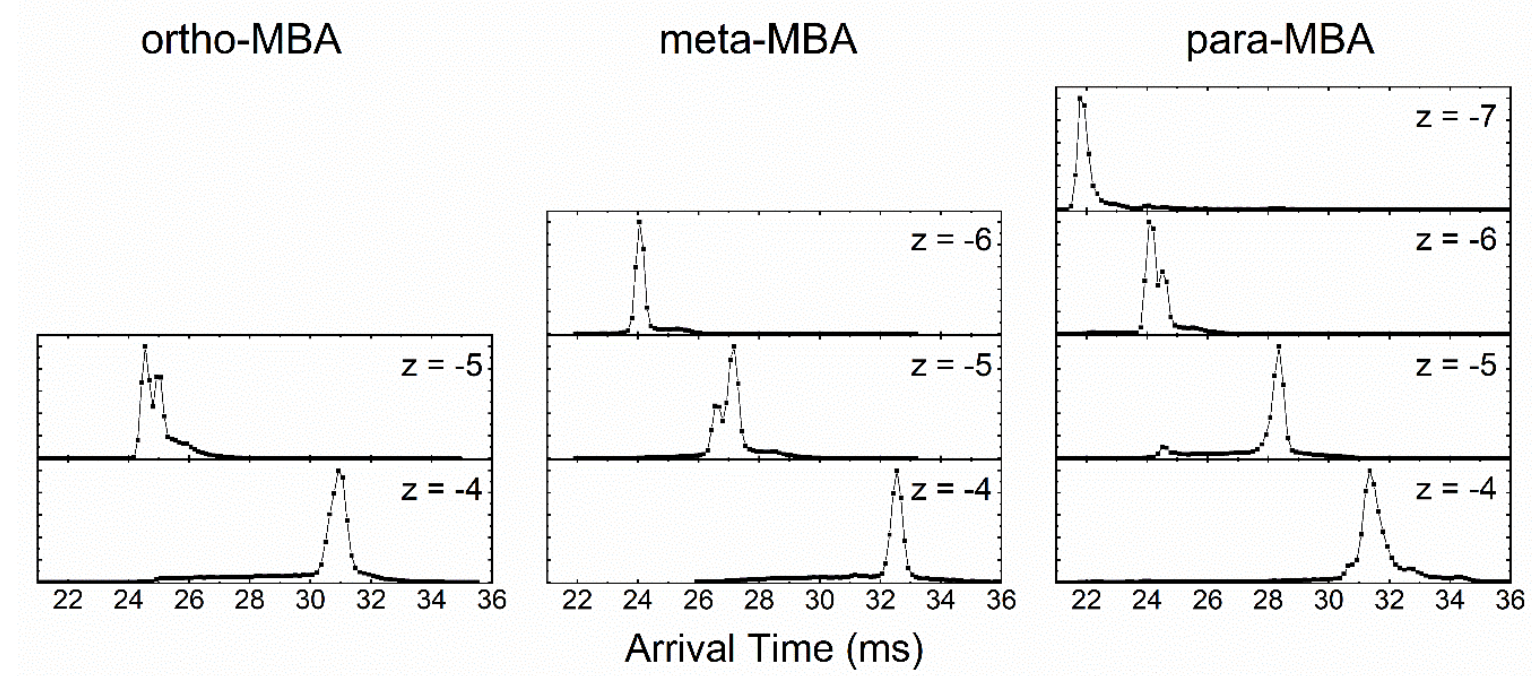

Figure 11: High-resolution arrival time distributions obtained in dual-drift tube configuration by integrating over the full isotope pattern for each charge state. Drift voltage $=1000 \mathrm{~V}$, drift length $=1.6 \mathrm{~m}$, drift gas: He, drift pressure: 4.0 Torr, drift temperature: $298 \mathrm{~K}$. 

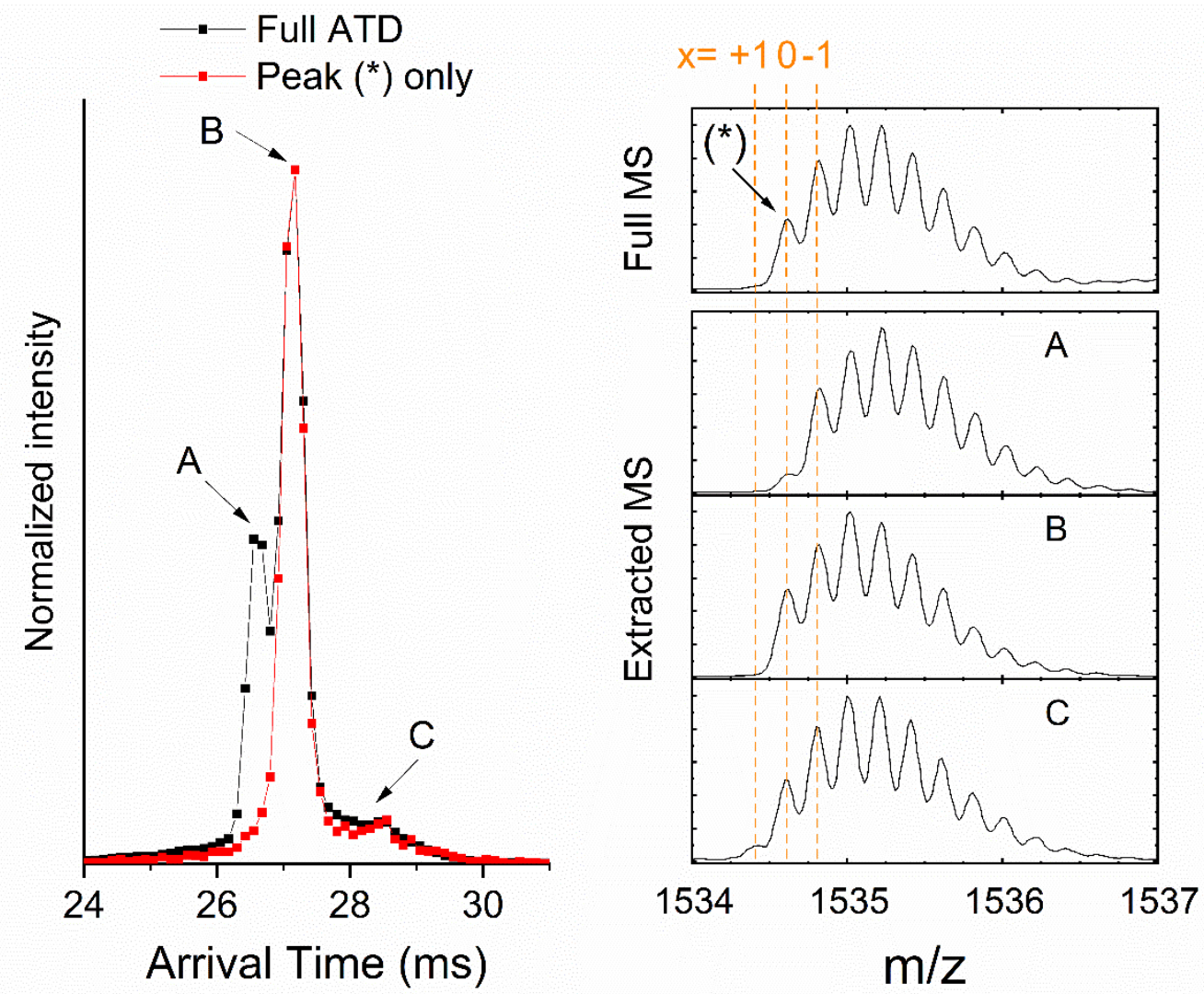

Figure 12: (Left) Extracted ATDs for $\left[\mathrm{Au}_{25} \mathrm{meta}-\mathrm{MBA}_{18}\right]^{-5}$ clusters from the full isotopic pattern (black), or (red) for the first isotopic peak visible at $\mathrm{m} / \mathrm{z} 1534.6$ $\left.{ }^{*}\right)$. (Right) Full mass spectrum and extracted mass spectra around $\mathrm{m} / \mathrm{z} 1535$. The extracted mass spectra are labelled $A, B$, and $C$ in relation with the selected arrival time extraction windows, corresponding to the different spectral features in the ATD. The dashed lines indicate the monoisotopic masses of different deprotonation patterns corresponding to different charge shifts $x$ ranging from -1 to +1 , and a total charge $z=-5$. 


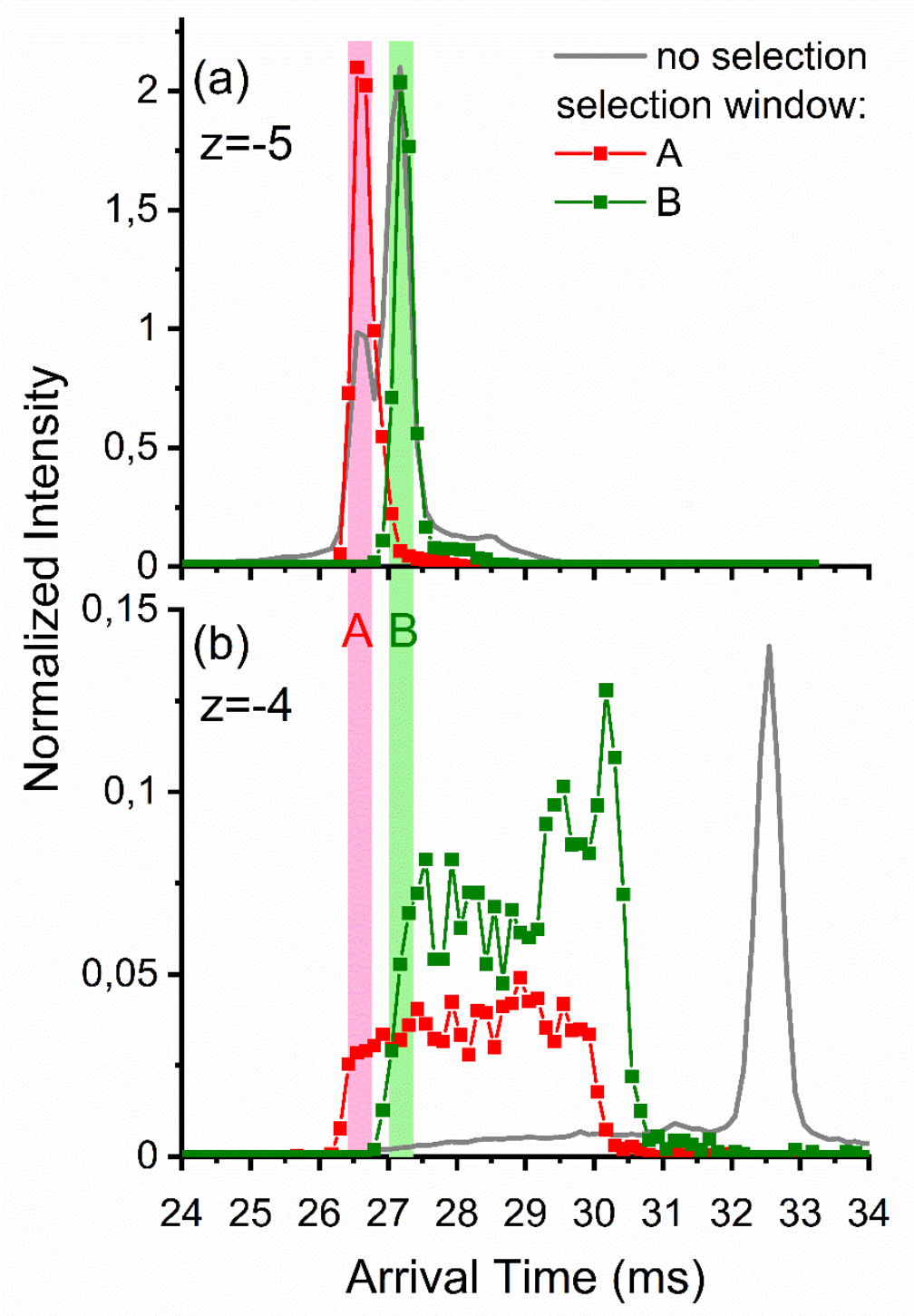

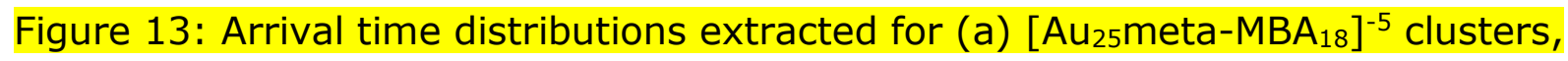
and (b) $\left[\mathrm{Au}_{25} \text { meta-MBA }{ }_{18}\right]^{-4}$ clusters, by integrating over the full isotopic pattern in each case. Red and green traces were obtained after selection of the ions on the fly (between the two drift tubes), respectively using the selection windows labelled A and B. These ATDs were normalized to the total ion signal (see text). The corresponding ATDs obtained without selection are also provided as a reference (grey lines). 


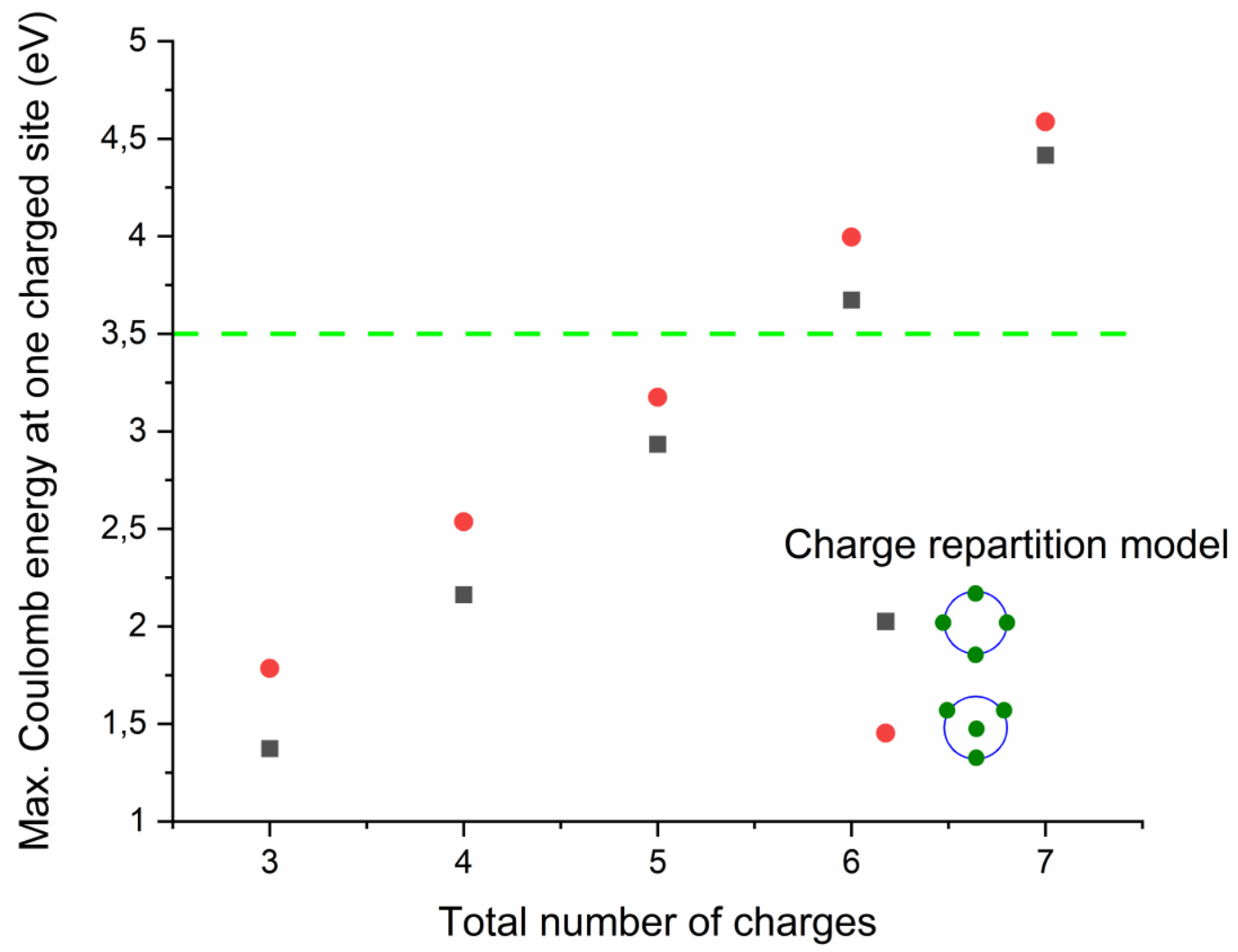

Figure 14: Coulomb repulsion energies calculated for charges constrained on a sphere with a radius comparable to that of the clusters. The reported values correspond to the maximum Coulomb energy at one charged site on the surface of the sphere. Two repartition models were considered: all charges on the surface of the sphere (black squares), or one charge at the center (red circles). The green dotted line represents a typical value for the binding energy of an electron on a carboxylate, taken from ref. 45. 


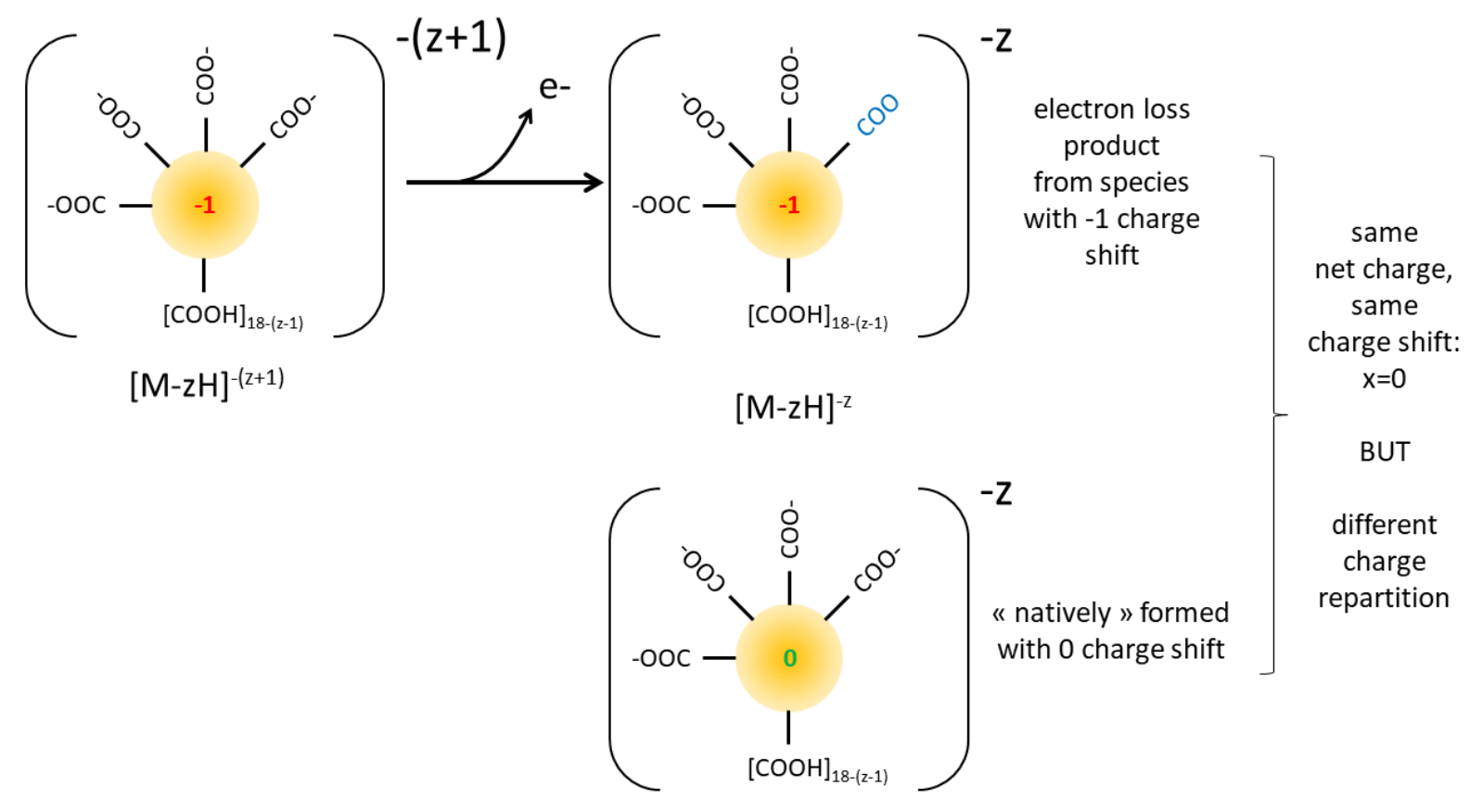

Scheme 1: Illustration of the impact of electron loss on the heterogeneity of clusters sharing the same apparent charging pattern. For simplicity, the charge shift was labelled at the center of the cluster. 


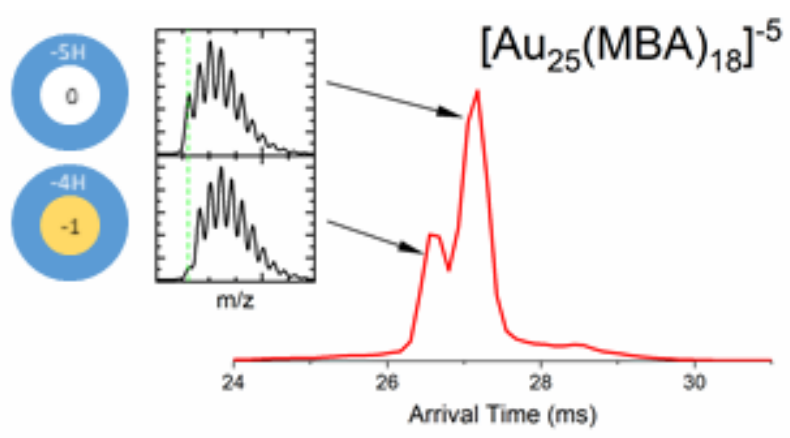

TOC Graphics 